\title{
Chalcones: Synthetic Chemistry Follows Where Nature Leads
}

\author{
Hiba A. Jasim ${ }^{1,2}{ }^{\mathbb{D}}$, Lutfun Nahar ${ }^{3, *} \mathbb{D}$, Mohammad A. Jasim ${ }^{4}\left(\mathbb{D}\right.$, Sharon A. Moore ${ }^{5} \mathbb{D}_{\text {, Kenneth J. Ritchie }}{ }^{1, *}$ \\ and Satyajit D. Sarker ${ }^{1}$ (iD
}

1 Centre for Natural Products Discovery (CNPD), School of Pharmacy and Biomolecular Sciences, Liverpool John Moores University, James Parsons Building, Byrom Street, Liverpool L3 3AF, UK; h.a.jasim@uoanbar.edu.iq (H.A.J.); S.Sarker@ljmu.ac.uk (S.D.S.)

2 Department of Biology, College of Education for Pure Sciences, University of Anbar, Al-Anbar 10081, Iraq

3 Laboratory of Growth Regulators, Institute of Experimental Botany ASCR \& Palacký University, Šlechtitelů 27, 78371 Olomouc, Czech Republic

4 Department of Biology, College of Education for Women, University of Anbar, Al-Anbar 10081, Iraq; mohammed.a.jasim@uoanbar.edu.iq

5 Faculty of Science and Engineering, University of Wolverhampton, Wolverhampton WV1 1LY, UK; sharonmoore1@hotmail.com

* Correspondence: drnahar@live.co.uk (L.N.); K.J.Ritchie@ljmu.ac.uk (K.J.R.); Tel.: +44-(0)-1512312219 (K.J.R.)

Citation: Jasim, H.A.; Nahar, L.; Jasim, M.A.; Moore, S.A.; Ritchie, K.J.; Sarker, S.D. Chalcones: Synthetic Chemistry Follows Where Nature Leads. Biomolecules 2021, 11, 1203. https: / / doi.org/10.3390/ biom 11081203

Academic Editor: Vladimir N. Uversky

Received: 31 July 2021

Accepted: 11 August 2021

Published: 13 August 2021

Publisher's Note: MDPI stays neutral with regard to jurisdictional claims in published maps and institutional affiliations.

Copyright: (c) 2021 by the authors. Licensee MDPI, Basel, Switzerland. This article is an open access article distributed under the terms and conditions of the Creative Commons Attribution (CC BY) license (https:// creativecommons.org/licenses/by/ $4.0 /)$.

\begin{abstract}
Chalcones belong to the flavonoid class of phenolic compounds. They form one of the largest groups of bioactive natural products. The potential anticancer, anti-inflammatory, antimicrobial, antioxidant, and antiparasitic properties of naturally occurring chalcones, and their unique chemical structural features inspired the synthesis of numerous chalcone derivatives. In fact, structural features of chalcones are easy to construct from simple aromatic compounds, and it is convenient to perform structural modifications to generate functionalized chalcone derivatives. Many of these synthetic analogs were shown to possess similar bioactivities as their natural counterparts, but often with an enhanced potency and reduced toxicity. This review article aims to demonstrate how bioinspired synthesis of chalcone derivatives can potentially introduce a new chemical space for exploitation for new drug discovery, justifying the title of this article. However, the focus remains on critical appraisal of synthesized chalcones and their derivatives for their bioactivities, linking to their interactions at the biomolecular level where appropriate, and revealing their possible mechanisms of action.
\end{abstract}

Keywords: chalcones; biomolecular interactions; synthesis; natural products; bioactivities; mechanisms; anticancer; antimicrobial; phenolics

\section{Introduction}

Chalcones are flavonoid-type phenolic phytochemicals, often referred to as 'openchain flavonoids', and biosynthesized via the shikimate pathway [1]. Chalcones are considered as the biosynthetic precursors of flavonoids. Chemically, chalcones are generally $\alpha, \beta$-unsaturated ketones consisting of two aromatic rings (rings A and B) linked through a three-carbon alkenone unit (Figure 1), but these may also include some saturated ketones, commonly known as dihydrochalcones, where instead of a three-carbon alkenone unit, a three-carbon alkanone unit is present. Among the naturally occurring chalcones, the presence of one or more phenolic hydroxyl functionalities is ubiquitous, and prenyl and geranyl substitutions on the aromatic rings are also widely observed. There are several thousand naturally occurring chalcones reported in the literature [2], and many of those chalcones were shown to interact with various biomolecules and possess cytoprotective and modulatory properties, making them potentially suitable candidates for therapeutic interventions in many human ailments. Several patents are also available for chalcones and their derivatives for their activities as anticancer, anti-inflammatory, antimitotic, antioxidant, and cytotoxic properties [3]. 
<smiles>O=C(C=Cc1ccccc1)c1ccccc1</smiles><smiles>O=C(C=Cc1ccccc1)c1ccccc1</smiles>

or

Figure 1. General structure of chalcones.

Because of structural simplicity and therapeutic potential, several bioinspired syntheses of chalcone derivatives and evaluation of their bioactivities were reported in the literature [2]. The first few attempts for the synthesis of chalcones began in the 1800s and continued through the subsequent centuries [4]. In this review article, some of these synthesized chalcones and their derivatives were critically appraised for their bioactivities, linking to their interactions at the biomolecular level where appropriate, and revealing their possible mechanisms of action. Moreover, this review article fundamentally aims to demonstrate how bioinspired synthesis of chalcone derivatives can potentially introduce a new chemical space for exploitation for new drug discovery, justifying the title of this article.

\section{Chalcone Synthesis}

Chalcones are considered privileged structures in the medicinal chemistry due to the relative ease with which they may be produced and modified, reflecting the similar ease with which they are produced in nature. From as early as the 19th century, many researchers have developed synthetic chalcones, with Kostanecki and Tambor being acknowledged as the first to successfully prepare synthetic chalcones using a method involving treatment of $o$-acetoxychalcone dibromides with alcoholic alkali $[5,6]$. However, the current methods of chalcone synthesis utilize an alkaline base and a polar solvent to couple two compounds with an aromatic ring each, e.g., acetophenone and benzaldehyde, and to produce the core chalcone nucleus (Scheme 1) [7-9]. Various aromatic compounds and different methods used in the synthesis of several chalcone derivatives (2-10) and chalcone itself (1) are summarized in Table 1.<smiles>O=Cc1ccccc1</smiles>

Acetophenone Benzaldehyde<smiles>CCO[C@H](O)c1ccc(C(=O)/C=C/c2ccccc2)cc1</smiles>

Chalcone

Scheme 1. Synthesis of chalcone.

Table 1. Synthesis of some chalcone and chalcone derivatives [7-9].

\begin{tabular}{|c|c|c|c|c|}
\hline Approach & Compound 1 & Compound 2 & $\begin{array}{l}\text { Reaction } \\
\text { Conditions }\end{array}$ & $\begin{array}{c}\text { Synthesize Chalcone and Its } \\
\text { Derivatives }\end{array}$ \\
\hline \multirow[t]{2}{*}{ Microwave } & & & $\begin{array}{l}\mathrm{NaOH} / \mathrm{MeOH} \text {, } \\
\text { microwave radiation } \\
\text { at } 110 \mathrm{~W}, 55^{\circ} \mathrm{C}\end{array}$ & \multirow[b]{3}{*}{ (1) } \\
\hline & & & $\begin{array}{l}\mathrm{I}_{2}-\mathrm{Al}_{2} \mathrm{O}_{3}, \text { microwave } \\
\text { radiation for } 80 \mathrm{~s}\end{array}$ & \\
\hline Ionic liquids & & & $\begin{array}{l}\mathrm{HBr}, \mathrm{Bmim}(\mathrm{OTs}) \\
12 \mathrm{~h}, 100{ }^{\circ} \mathrm{C}\end{array}$ & \\
\hline
\end{tabular}


Table 1. Cont.

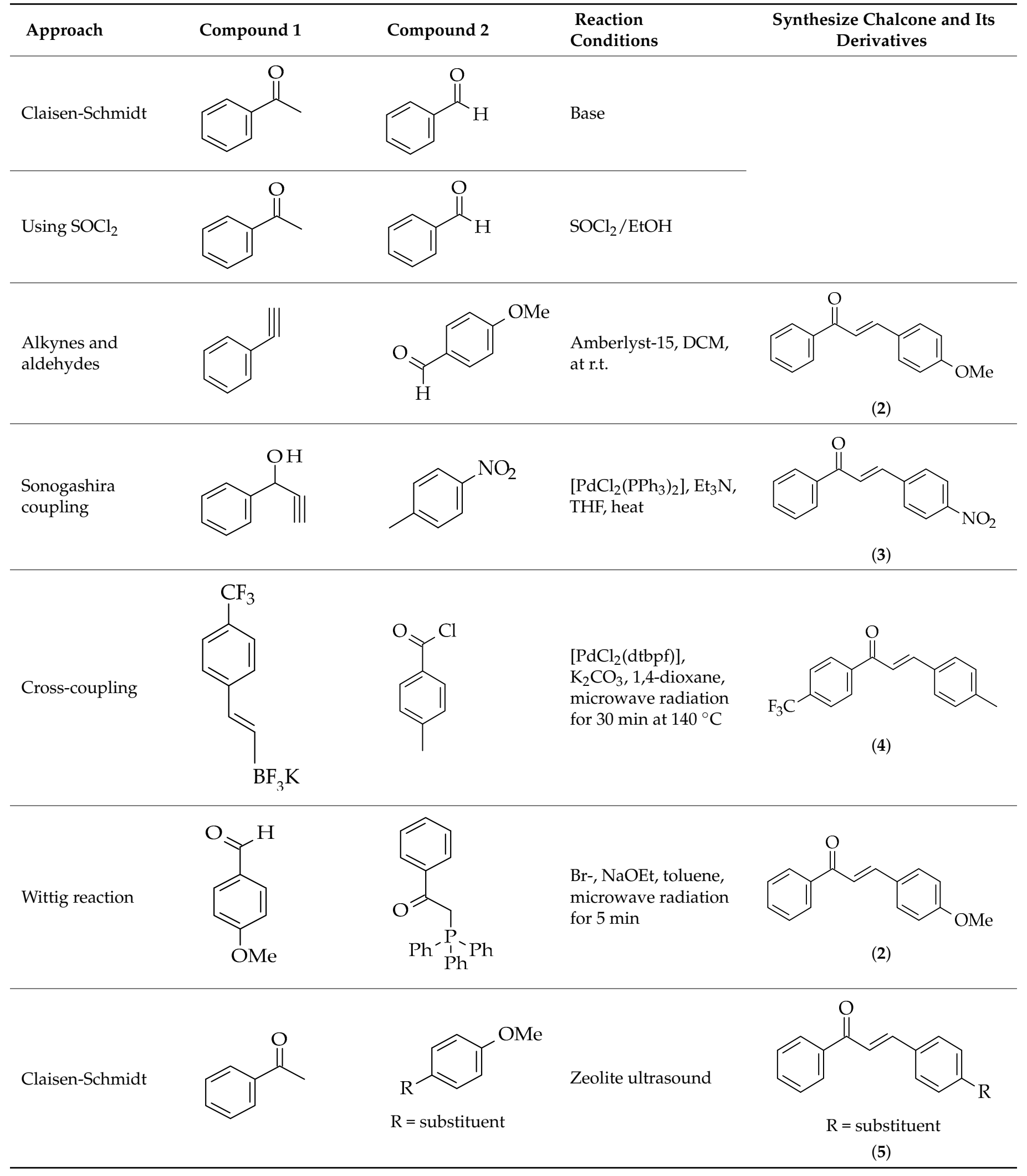


Table 1. Cont.

Compound 12 Compound 2<smiles>[R]c1cc(C(=O)Cl)cc([R])c1[R]</smiles><smiles>OB(O)/C=C/c1ccccc1</smiles><smiles>[R]c1cc(C(=O)/C=C/c2ccccc2)cc([R])c1[R]</smiles>

(8)

Suzuki reaction<smiles>O=C(OC(=O)c1ccccc1/C=C/B(O)/C=C/c1ccccc1)c1ccccc1</smiles><smiles>O=C(/C=C/c1ccccc1)c1ccccc1</smiles>

(1)<smiles>OB(O)c1ccccc1</smiles><smiles>CC(=O)/C=C/c1ccccc1</smiles>

Pd<smiles>O=C(/C=C/c1ccccc1)c1ccccc1</smiles>

(1)

Base-driven<smiles>CC(=O)c1ccc(O)cc1O</smiles>
$\mathrm{HO}$<smiles>O=Cc1ccc(O)cc1</smiles>
1-5 h<smiles>CC(=O)c1c(C)cc(O)cc1O</smiles><smiles>COc1cc(O)cc(OC)c1C(C)=O</smiles>

$\mathrm{NaOH}$<smiles>O=C(/C=C/c1ccc(O)cc1)c1ccc(O)cc1O</smiles>

(9)<smiles>COc1ccc(/C=C/C(=O)c2c(OC)cc(O)cc2OC)cc1</smiles>

(10)

$\mathrm{Al}_{2} \mathrm{O}_{3}$ : aluminum oxide; Bmim (OTs): 1-butyl-3-methyl-1H-imidazolium 4-methylbenzenesulfonate; DCM: dichloromethane; EtOH: ethanol; $\mathrm{Et}_{3} \mathrm{~N}$ : triethylamine; $\mathrm{NaOEt}$ : sodium ethoxide; $\mathrm{HBr}$ : hydrobromic acid; $\mathrm{H}_{2} \mathrm{SO}_{4}$ : sulfuric acid; $\mathrm{K}_{2} \mathrm{CO}_{3}$ : potassium carbonate; $\mathrm{KOH}$ : potassium hydroxide; $\mathrm{MeOH}$ : methanol; $\mathrm{Na}_{2} \mathrm{CO}_{3}$ : sodium carbonate; $\mathrm{NaOH}$ : sodium hydroxide; $\mathrm{Pd}$ : palladium; $\mathrm{PdCl}_{2}$ : palladium chloride; $\mathrm{PdCl}_{2}\left(\mathrm{PPh}_{3}\right)_{2}$ : bis(triphenylphospine)palladium dichloride; $\mathrm{PdCl}_{2}(\mathrm{dtbpf})$ : 1,1'-bis(di-tert-butylphosphino)ferrocene]dichloropalladium; $\left(\mathrm{PPh}_{3}\right)_{4} \mathrm{Pd}^{(0)}$ : tetrakis(triphenylphosphine)palladium(0); $\mathrm{SiO}_{2}$ : silicon dioxide; $\mathrm{SOCl}_{2}$ : thionyl chloride; THF: tetrahydrofuran. 


\section{Molecular Mechanisms of Chalcone Actions}

Various bioactivities of chalcones result from their interactions with several biomolecules and different signaling pathways. Some of those molecular mechanisms of activities of chalcones are discussed in the following subsections.

\subsection{Activation of Nuclear Factor-Erythroid 2 p45 Subunit-Related Factor 2 Pathway and Cellular Defence Genes}

Nuclear factor-erythroid (NF-E2) p45-related factor 2 (Nrf2) is a transcription factor, which controls the expression of a battery of antioxidant genes, all of which have an antioxidant response element (ARE) in their promoter which allows the binding of Nrf2 [10]. The ARE-Nrf2 complex leads to induction of cell expression of cell defense genes. such as glutathione S-transferase (GST), hemoxygenase-1 (HO-1), and xenobiotic metabolizing enzyme (NAD(P)H: quinone oxidoreductase 1) (NQO1) [11-13]. Kim et al. [14] synthesized a series of chalcone derivatives, and among them, a new chalcone derivative (11) (Figure 2) was found to possess the best Nrf2 activation properties and could induce Nrf2 nuclear translocation. This chalcone salt (11) was shown to induce gene expression of antioxidant enzymes and attenuate inflammatory responses in activated microglia. There has been an increasing number of publications that detail the relationship between chalcones and Nrf2, with several studies reporting the ability of chalcones to induce Nrf2. The ability of synthetic and natural chalcones to activate the Nrf2 signaling pathway was reviewed recently [15].<smiles>CN1CCN(C(=O)COc2ccc(C(=O)/C=C/c3ccccc3C(F)(F)F)cc2)CC1</smiles>

(11)

Figure 2. A synthetic chalcone derivative as an Nrf2 activator.

Ajiboye et al. [16] reported that natural chalcones lophirones B (12) and C (13) could induce Nrf2 (Figure 3). In addition, the natural chalcone, 3,4,2', $4^{\prime}$-tetrahydroxychalcone $4^{\prime}$-O- $\beta$-D-glucopyranoside (14), was noted to increase the level of NQO1 [NAD(P) H: quinone oxidoreductase 1] in Hepa 1c1c7 cells [17]. Martinez et al. [18] reported that chalcone (1) could increase glutathione (GSH), HO-1, and Nrf2 (Figure 3). Furthermore, other researchers found 2-chloro- $4^{\prime}, 6^{\prime}$-dimethoxy-2'-hydroxychalcone (15), a synthetic chalcone, to increase GSH levels by enhancing its biosynthesis [19]. The ability to induce activation of Nrf2 was exploited to assess potential cancer chemopreventive activity of licorice samples collected from different geographical locations [20]. In fact, the structural diversity that exists among synthetic and natural chalcones, ease of structural modification, and the presence of $\alpha, \beta$-unsaturated carbonyl functionality (pharmacophore) make these compounds suitable drug candidates or can lead to targeting of Nrf2-dependent disease.

\subsection{Activation of the Nuclear Factor-Kappa B (NF-אB) PI3K/Akt Signaling Pathway}

The transcription factor, nuclear factor-kappa B PI3K/Akt (NF-kB), is a heterodimer consisting of p50 and p65 subunits, and is central to the functioning of the immune system. It has a role to play in the expression of immunoglobulin-k. NF- $\mathrm{BB}$ is activated by extracellular promoters, such as growth factors and inflammatory cytokines. The extracellular promoters switch on NF- $\kappa B$ signaling through dissolution and phosphorylation of the inhibiting I-KB protein. Allowing NF- $\mathrm{KB}$ to enter the nucleus and regulate cell cycle and apoptosis-related gene expression [21]. 


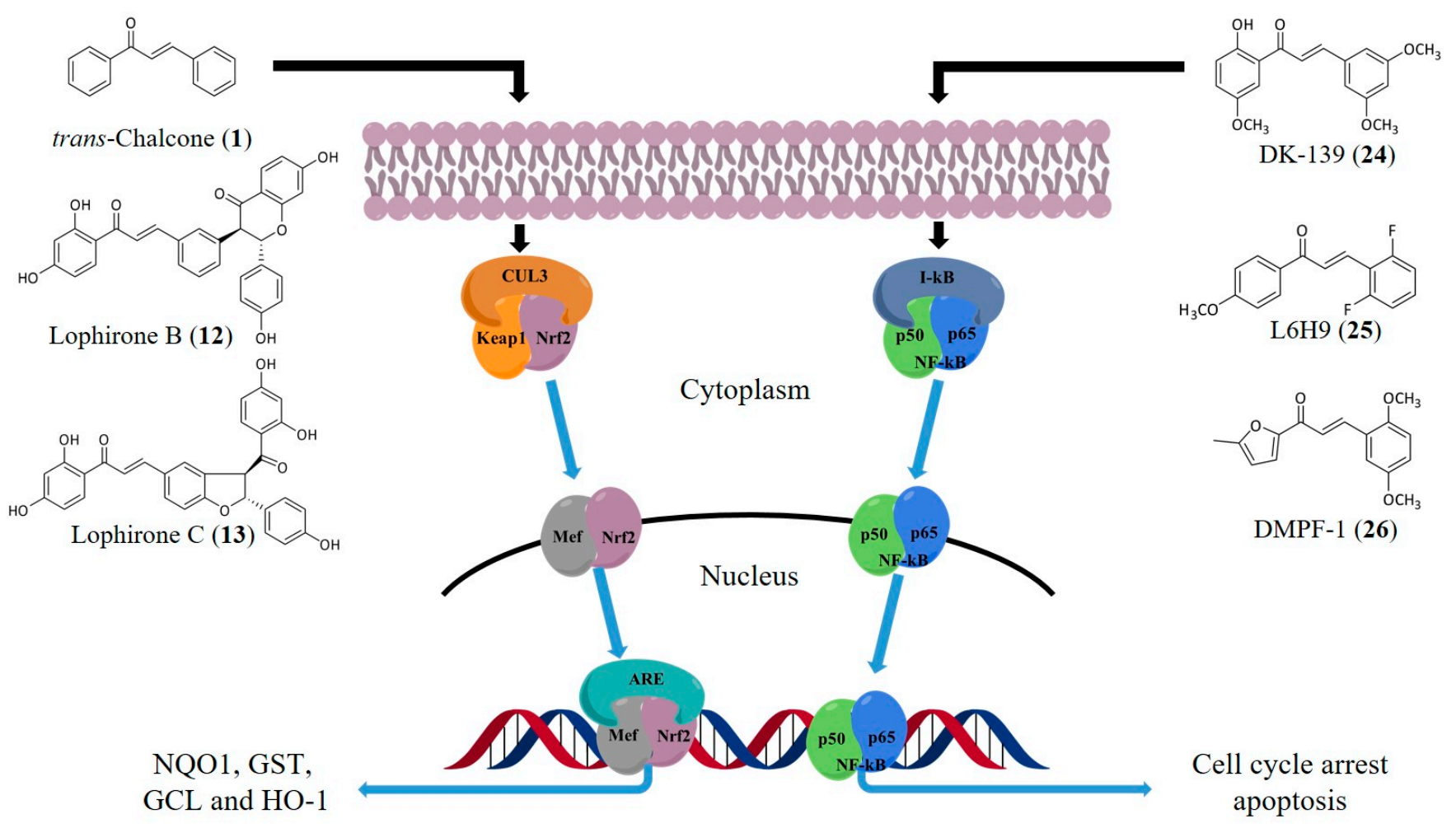

Figure 3. Chalcones that induce transcription factors: chalcone (1), lophirone B (12) and C (13) induce the Nrf2 pathway, while synthetic chalcones DK-139 (24), L6H9 (25) and DMPF-1 (26) induce the NF-kB pathway.

Several studies have examined the effect of chalcones on NF- $\kappa$ B signaling as a plausible way to modulate inflammation and cancer, and found that several natural chalcones, e.g., butein (16), calomelanone (17), flavokawain C (18), homobutein (19), 4-hydroxychalcone (20), 4'-hydroxychalcone (21), isoliquiritigenin (22), 4-methoxychalcone (2), and phloretin (23) (Figure 4) have the ability to suppress NF-kB signaling [22]. Similarly, NF-kB activation could be stimulated by several synthetic chalcones, such as 2-hydroxy- $3^{\prime}, 5,5^{\prime}$ trimethoxychalcone (DK-139) (24) [23], (E)-2,6-difluoro-4'-methoxychalcone (L6H9) (25) [24], and 3-(2,5-dimethoxyphenyl)-1-(5-methylfuran-2-yl)prop-2-en-1-one (DMPF-1) (26) [25] (Figure 3).<smiles>[R]Oc1cc(/C=C/C(=O)c2ccc(O)cc2O)ccc1O</smiles>

Butein (16) $\mathrm{R}=\mathrm{H}$

Homobutein (19) $\mathrm{R}=\mathrm{Me}$<smiles>[R]Oc1ccc(/C=C/C(=O)c2ccccc2)cc1</smiles>

4-Hydroxychalcone (20) $\mathrm{R}=\mathrm{H}$ 4-Methoxychalcone (2) $\mathrm{R}=\mathrm{Me}$<smiles>[R20]Oc1ccc(/C=C/C(=O)c2c(O)cc([R20])cc2O[Z20])cc1</smiles>

Calomelanone (17) $\mathrm{R}=\mathrm{R}^{\prime}=\mathrm{Me} ; \mathrm{R}^{\prime \prime}=\mathrm{H}$

Flavokawain $C(\mathbf{1 8}) \mathrm{R}=\mathrm{R}^{\prime}=\mathrm{R}^{\prime \prime}=\mathrm{Me}$

Phloretin (23) $\mathrm{R}=\mathrm{R}^{\prime}=\mathrm{R}^{\prime \prime}=\mathrm{H}$

Figure 4. Examples of some naturally occurring chalcones that induce the NF- $\mathrm{kB}$ pathway. 


\subsection{Effects on the Cell Cycle}

Many researchers have investigated the effects of synthetic and natural chalcones on the cell cycle [26-30]. Dimethyl-cardamonin (27) was shown to arrest cell cycle in the $\mathrm{G}_{1}$ phase by decreased expression of cyclin D1, CDK4 (cyclin-dependent kinase 4), and phospho- $\mathrm{Rb}$ [30], while Hseu et al. [29] reported that the chalcone flavokawain B (28) could cause cell cycle arrest in the $\mathrm{G}_{2} / \mathrm{M}$ phase in human oral carcinoma cells.

Studies conducted by Maioral et al. [31] established the importance of chalcone (2E)-1(2,5-dimethoxy-phenyl)-3-(1-naphthyl)-2-propene-1-one (29) in blocking the $\mathrm{G}_{2} / \mathrm{M}$ phase in human leukemia cell lines, i.e., NB4, K562, and Kasumi cell lines, and the $G_{0} / G_{1}$ phase in the human T-cell leukemia cell line CEM, the human lung cancer cell line U937, and the $S$ phase in Jurkat cell lines. In addition, Kello et al. [32] found that the chalcones (E)-2(4'-methoxybenzylidene)-1-benzosuberone (30) and (E)-2-( $3^{\prime}, 4^{\prime}$-dimethoxy-benzylidene)1-tetralone (31) could be responsible for cell cycle arrest at the $\mathrm{G}_{2} / \mathrm{M}$ phase in Caco-2 cells. Two synthetic chalcones, (E)-1-(4-aminophenyl)-3-(2,3-dimethoxyphenyl)-prop-2-en1-one (32) and (E)-1-(4-aminophenyl)-3-phenylprop-2-en-1-one) (33), were studied for their effects on the cell cycle of the human erythroleukemic cell line K562 and it was observed that both chalcones acted as inhibitors of the cell cycle [33]. The cell cycle distribution of cervical cancer (HeLa) cells treated with the synthetic chalcone L1 (39) was analyzed by flow cytometry, revealing its ability to induce cell cycle arrest in the $\mathrm{G}_{2} / \mathrm{M}$ phase after $24 \mathrm{~h}$ of treatment [26], while chalcone (1) and licochalcone A (40) were found to induce the cell cycle arrest in the $G_{1}$ phase in the MCF-7 breast cancer cells [27]. A summary of the effects of different chalcones on the cell cycle is shown in Figure 5, and the structures of chalones that influence the cell cycle are presented in Figure 6.

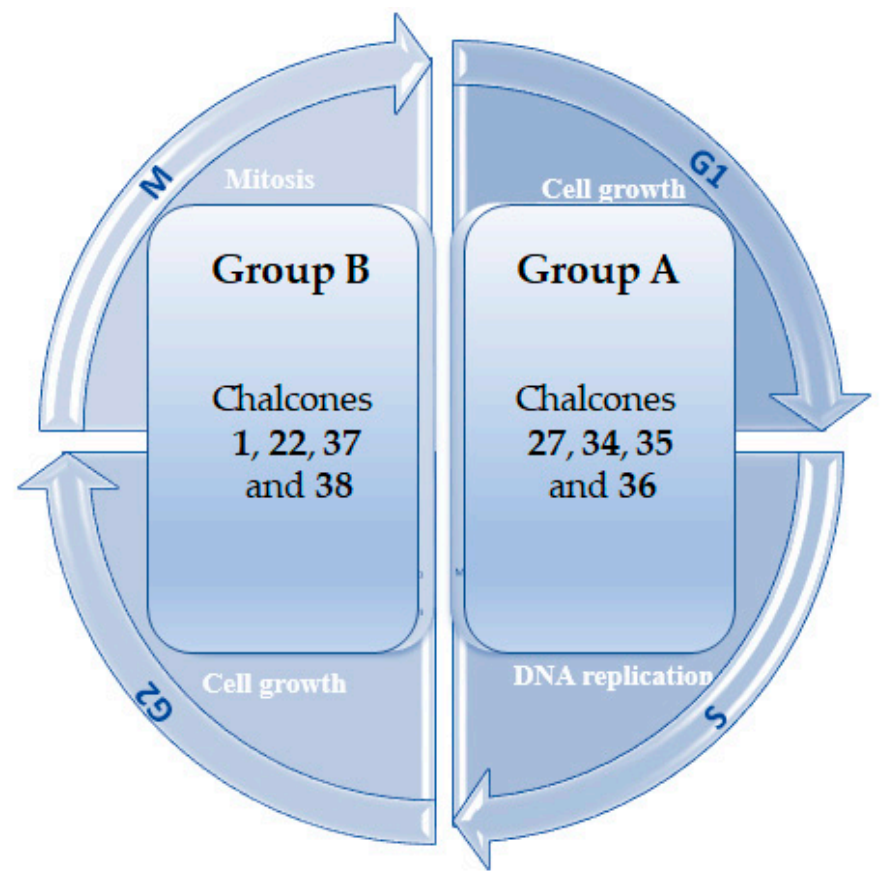

Figure 5. The effect of natural and synthetic chalcones on the cell cycle. Group A: Chalcones arrest the cell cycle at the $\mathrm{G}_{1} / \mathrm{S}$ phase, i.e., dimethyl cardamonin (27) [30], tetramethoxychalcone (34) [28], 2'-hydroxy-2,3,4', $6^{\prime}$-tetramethoxychalcone (35) [34], and tetrahydro-[1,2,4]triazolo[3,4-a]isoquinoline chalcones (36) [35]. Group B: Chalcones that arrest the cell cycle at the $\mathrm{G}_{2} / \mathrm{M}$ phase chalcone (1) [29], isoliquiritigenin (22) [36], (2E)-3-(acridin-9-yl)-1-(2,6-dimethoxyphenyl)prop-2-en-1-one (37) [37], and quinazolinone-chalcone (38) [38]. 
<smiles>COc1cccc(OC)c1C(=O)/C=C/c1c2ccccc2cc2ccccc12</smiles>

(2E)-3-(Acridin-9-yl)-1-(2,6-dimethoxyphenyl)prop-2-en-1-one<smiles>[R]c1cccc(/C=C/C(=O)c2ccc(N)cc2)c1[R]</smiles>

(E)-1-(4-Aminophenyl)-3-(2,3-dimethoxyphenyl)-prop-2-en-1-one (32)

$$
\mathrm{R}=\mathrm{R}^{\prime}=\mathrm{OMe}
$$

(E)-1-(4-Aminophenyl)-3-phenylprop-2-en-1-one) (33) $\mathrm{R}=\mathrm{R}^{\prime}=\mathrm{H}$<smiles>COc1ccc(OC)c(C(=O)/C=C/c2cccc3ccccc23)c1</smiles>

(2E)-1-(2,5-Dimethoxy-phenyl)-3-(1-naphthyl)-2-propene-1-one

(29)<smiles>COc1c(C)c(O)c(C)c(O)c1C(=O)/C=C/c1ccccc1</smiles>

Dimethyl cardamonin (27)<smiles>COc1cc(O)c(C(=O)/C=C/c2ccccc2)c(OC)c1</smiles>

Flavokawain B (28)<smiles>COc1ccc(C=C2CCc3ccccc3C2=O)cc1OC</smiles>

(E)-2-(3',4'-Dimethoxy-benzylidene)-1-tetralone<smiles>COc1cc(O)c(C(=O)/C=C/c2cccc(OC)c2OC)c(OC)c1</smiles><smiles>COc1cc(OC)c(/C=C/C(=O)c2cn(OC)c3ccccc23)c(OC)c1</smiles>

L1 (39)<smiles>COc1ccc(/C=C2\CCCc3ccccc3C2=O)cc1</smiles>

(E)-2-(4'-Methoxybenzylidene)-1-benzosuberone (30)<smiles>COc1cc2c(cc1OC)C1N(CC2)C(C(=O)/C=C/c2cc(OC)c(OC)c(OC)c2)=NN1c1ccccc1</smiles>

Tetrahydro-[1,2,4]triazolo[3,4-a]isoquinoline chalcones (36)
2'-Hydroxy-2,3,4',6'-tetramethoxychalcone (35)<smiles>C=CC(C)(C)c1cc(/C=C/C(=O)c2ccc(O)cc2)c(OC)cc1O</smiles>

Licochalcone A (40)<smiles>COc1cc(/C=C/C(=O)c2cccc(-n3c(C)nc4ccccc4c3=O)c2)cc(OC)c1OC</smiles>

Quinazolinone-chalcone (38)<smiles>COc1ccc(/C=C/C(=O)c2cc(OC)c(OC)c(OC)c2)cc1</smiles>

Tetramethoxychalcone (34)

Figure 6. Structures of some chalcones that interfere with the cell cycle.

\subsection{Effects on Apoptosis}

Apoptosis is a form of programmed cell death in multicellular organisms, governed by a series of biochemical events [39]. Several studies have examined the effect of chalcones on apoptosis to establish whether chalcones induce cell death through apoptosis or necrosis. 
One of the early studies reported that chalcones (1) could induce apoptosis through enhancing the pro-apoptotic molecules, Bax and Bak, whilst reducing anti-apoptotic Bcl proteins (Figure 7) [40]. Chen et al. [41] demonstrated that the natural chalcone lonchocarpin (41) decreased cell proliferation via activation of caspase-3, caspase-9, and Bax (Figure 7). The synthetic chalcone, (2E)-3-(2-naphthyl)-1-(3'-methoxy-4'-hydroxy-phenyl)-2-propen-1-one (42), could block the $\mathrm{G} 2 / \mathrm{M}$ phase, in addition to increasing p53 and Bax expression, thus bringing about caspase-3 activation and cell death [42].

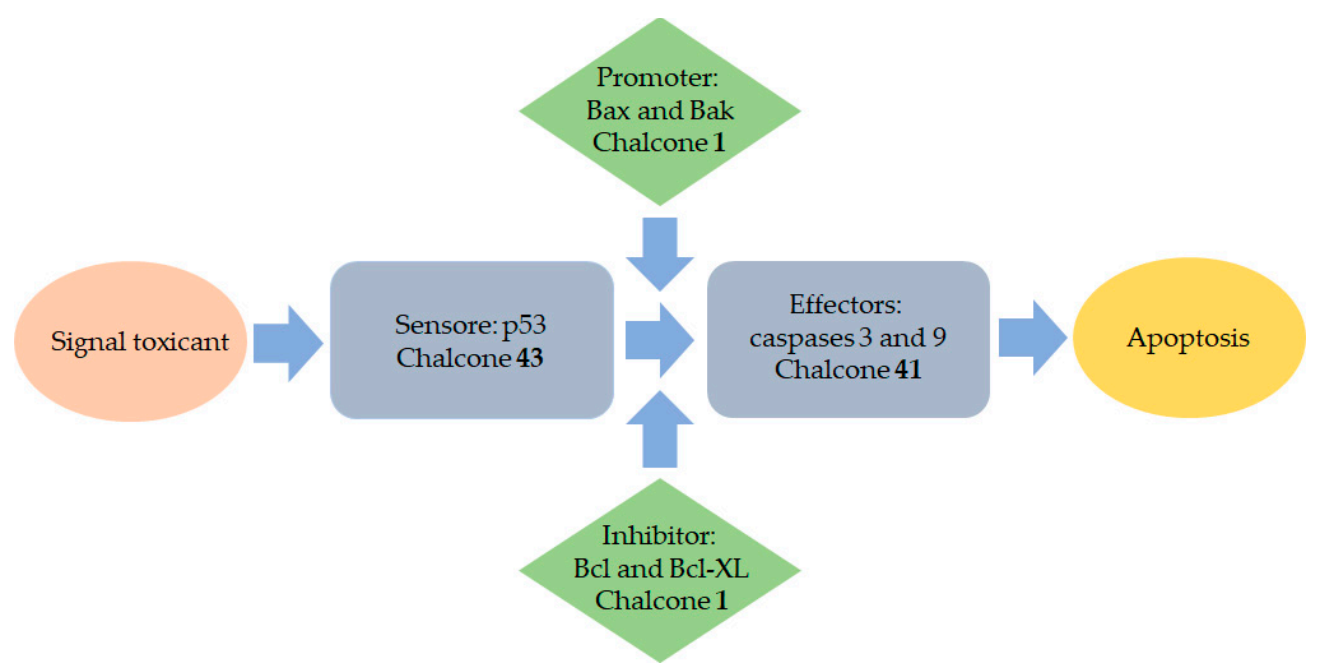

Figure 7. Schematic diagram showing how chalcones induce apoptosis.

Furthermore, it was shown that a synthetic chalcone (2E)-1-(2,5-dimethoxy-phenyl)3-(1-naphthyl)-2-propene-1-one (29) could induce cell death in human acute leukemia cell lines [31]. A recent study by Novilla et al., (2017) pointed out that synthetic chalcones (E)-1-(4-aminophenyl)-3-(2,3-dimethoxy-phenyl)-prop-2-en-1-one (32) and (E)-1-(4aminophenyl)-3-phenylprop-2-en-1-one) (33) could induce expression of caspase-3 in K562 cells. Additionally, another synthetic chalcone derivative, (E)-3-(4-methoxyphenyl)-2methyl-1-(3,4,5-trimethoxyphenyl)prop-2-en-1-one (43), was proposed as useful against prostate cancer as it was noted to cause cell cycle arrest and apoptosis (Figure 7) [43]. The structures of chalones that can influence apoptosis are presented in Figure 8.<smiles>CC1(C)C=Cc2c(ccc(C(=O)/C=C/c3ccccc3)c2O)O1</smiles>

Lonchocarpin (41)<smiles>COc1cc(C(=O)/C=C/c2ccc3ccccc3c2)ccc1O</smiles>

(2E)-3-(2-Naphthyl)-1-(3'-methoxy-4'-hydroxyphenyl)-2-propen-1-one (42)<smiles>COc1ccc(/C=C(\C)C(=O)c2cc(OC)c(OC)c(OC)c2)cc1</smiles>

(E)-3-(4-Methoxyphenyl)-2-methyl-1-(3,4,5-trimethoxyphenyl)prop-2-en-1-one (43)

Figure 8. Structures of some chalcones that influence apoptosis.

\subsection{Binding with the Estrogenic Receptor (ER)}

Chalcones, especially several natural chalcones present in food plants, are known to possess estrogenic properties, which are mediated by their interactions with the estrogenic 
receptor (ER) [44,45]. Isoliquiritigenin (22), a natural chalcone that is usually found in licorice, was shown to have estrogen receptor $\alpha$-dependent growth-promoting effects on breast cancer cells [44]; its estrogenic effect was established using the hormone-sensitive MCF-7 breast cancer and steroid-independent HeLa cells. This chalcone was found to transactivate the endogenous estrogen receptor $\alpha$ in MCF-7 cells, supported by its ability to induce the down-regulation of estrogen receptor $\alpha$ protein levels and the up-regulation of pS2 mRNA. Furthermore, it was shown that this chalcone could exert agonistic effect for both estrogen receptor isoforms. In silico studies performed with the chalcone $2^{\prime}, 4^{\prime}$ dihydroxy-6-methoxy-3,5-dimethylchalcone (44) (Figure 9), a component of the leaves of Eugenia aquea established its interaction with estrogen receptor $\alpha$ [45,46]. Another similar virtual screening of $2^{\prime}, 3^{\prime}, 4^{\prime}$-trihydroxychalcone (45) showed that this chalcone acted as an estrogen receptor ligand that could modulate the activity of $17 \beta$-estradiol [47].<smiles>[C+]C=C(C([CH+])=O)C(=CC(=O)c1ccc(OCCN(C)C)cc1)c1c(Cl)cccc1Cl</smiles><smiles>COc1c(C)cc(C)cc1/C=C/C(=O)c1ccc(O)cc1O</smiles><smiles>O=C(/C=C/c1ccccc1)c1ccc(O)c(O)c1O</smiles>

$2^{\prime}, 3^{\prime}, 4^{\prime}$-Trihydroxychalcone (45)<smiles>O=C(/C=C/c1ccc(O)cc1)c1ccc(O)cc1OCCO</smiles>

Figure 9. Examples of chalcones that bind to estrogenic receptors.

Branham et al. [48] used the estrogen receptor-ligand binding assay to assess the relative binding affinity of five chalcones, i.e., chalcone (1), 4-hydroxychalcone (20), 4' hydroxychalcone (21), phloretin (23), and 4,2', $4^{\prime}$-trihydroxychalcone (46), and a significant level of binding with the estrogen receptor $\alpha$ was observed with all these chalcones. In this study, a preliminary attempt was made to describe possible structure-activity relationships of the chalcones used in the study. Chalcone (1) was found to bind with the estrogen receptor with a weaker affinity than that of the monohydroxylated chalcones $\mathbf{2 0}$ and $\mathbf{2 1}$, whereas multiple hydroxylation, e.g., in chalcone 46, appeared to have decreased the binding affinity. Several chalcone-phenylpyran-2-one derivatives bearing a $N, N$-dimethyl ethylamine side chain, for example, chalcone 47 , were synthesized as estrogen receptor modulators [49]. All synthesized chalcone derivatives showed selectivity toward estrogen receptor $\alpha$, and among the compounds, the derivative with 2,6-dichloro functionalities (47) showed the strongest affinity toward the receptor.

\section{Bioactivity of Chalcones}

Chalcones are known as biologically active molecules. Different studies with natural and synthetic chalcones have demonstrated their bioactivities in vitro, in vivo, and most recently in silico. Major bioactivities of chalcones include their activities as anticancer, antidiabetic, antimicrobial, and antioxidant agents. Moreover, various chalcones were shown to possess antiparasitic properties. Most of their activities, particularly their anticancer potential, is associated with some of the biomolecular mechanisms outlined in Table 1 in this article. Bioactivities of chalcones are described with specific examples in 
the following subsections. Figure 10 presents the chemical structures of those bioactive chalcones, unless the structures are shown earlier in this article, whilst Table 2 summarizes various bioactivities of naturally occurring chalcones [50-81].<smiles>COc1cc(O)cc(O)c1C(=O)/C=C/c1ccccc1</smiles>

[2',4'-Dihydroxy-6'-methoxychalcone]<smiles>[R10]CC(=O)c1c(OC)cc(O)cc1C(=O)CCc1ccc(O)cc1</smiles>

$\mathrm{HO}$<smiles>O=C(/C=C/c1ccccc1)c1ccc(O)cc1O</smiles><smiles>COc1ccc(C(=O)/C=C/c2ccc(O)cc2)c2c1CCC(C)(C)O2</smiles>

Deoxydihydroxanthoangelol H (49)<smiles>COc1cc(O)c(/C=C/C(=O)c2ccccc2)c(O)c1</smiles>

2,6-Dihydroxy-4-methoxychalcone (52)

Dihydrochalcone diglycoside (50)<smiles>CC(C)=CCc1c(O)ccc(C(=O)/C=C/c2ccccc2)c1O</smiles>

(E)-1-(2,4-Dihydroxy-3-(3-methylbut-2-en-1-yl)phenyl)-3-phenylprop-2-en1-one (53)<smiles>[X]c1cc(O)c(C(=O)/C=C/c2ccc(OC)c([R])c2OC)c(OC)c1</smiles>

3,2'-Dihydroxy-2,4,4',6'-tetramethoxychalcone (55) $\mathrm{R}=\mathrm{OH}$

2'-Hydroxy-2,4,4',6'-tetramethoxychalcone (56) $\mathrm{R}=\mathrm{H}$

2'-Hydroxy-2,3,4,4',6'-pentamethoxychalcone (57)) $\mathrm{R}=\mathrm{OMe}$<smiles>COc1cc(O)ccc1/C=C/C(=O)c1ccc(O)cc1</smiles>

Echinantin (59)

[4,4'-Dihydroxy-6-methoxychalcone]<smiles>COc1cc(O)c(C(=O)/C=C/c2ccccc2)cc1OC</smiles>

2'-Hydroxy-4',5'-dimethoxychalcone (61)<smiles>COc1cc(O)c(C(=O)/C=C/c2ccccc2)c(O)c1</smiles>

2',6'-Dihydroxy-4'-methoxychalcone (54)<smiles>Cc1c(O)c2c(c(C(=O)/C=C/c3ccccc3)c1O)OC(C)(C)C=C2</smiles>

1-(5,7-Dihydroxy-2,2,6-trimethyl-2H-1-benzopyran-8-yl)-3-phenyl-2-propen-1-one [58]<smiles>COc1ccc(/C=C/C(=O)c2c(O)cc(OC)cc2OC)cc1</smiles>

Flavokawain A (60)

[2', 4, 4'-Trimethoxy-5'-hydroxychalcone]<smiles>[R20]c1ccc(C(=O)/C=C/c2ccc(O)cc2)c(O)c1CC=C(C)C</smiles>

4-Hydroxyderricin (62) $\mathrm{R}=\mathrm{Me}$ Isobavachalcone (63) $\mathrm{R}=\mathrm{H}$

Figure 10. Cont. 
<smiles>[R]c1ccc(/C=C/C(=O)c2c(O)c(Cc3c(O)c(C)c(O)c(C(C)=O)c3O)c(O)c3c2OC(C)(C)C=C3)cc1</smiles>

4'-Hydroxyrottlerin (64) $\mathrm{R}=\mathrm{OH}$

Rottlerin (65) $\mathrm{R}=\mathrm{H}$<smiles>CC(=O)c1c(O)c(C)c(O)c(Cc2c(O)c(Cc3c(O)c(C)c(O)c(C(C)=O)c3O)c3c(c2O)OC(C)(C)CC24CC(C)(C)Oc5c(C(=O)C=Cc6ccc(O)cc6)c(c(O)c(C=Cc6ccc(O)cc6)c52)C4(C)O3)c1O</smiles>

Kamalachalcone E (67)<smiles>COc1c(/C=C/C(=O)c2ccc(O)c(CC=C(C)C)c2)ccc(O)c1O</smiles><smiles>[R]c1cc(/C=C/C(=O)c2c(O)c(C/C=C(/C)CCC=C(C)C)c(O)c3c2OC(C)(C)C=C3)ccc1O</smiles>

Mallotophilippens C (74) $\mathrm{R}=\mathrm{H}$

Mallotophilippens D (75) $\mathrm{R}=\mathrm{OH}$<smiles>CC(O)C(O)COc1ccc(C(=O)/C=C/c2ccc(O)c(O)c2)c(O)c1O</smiles><smiles>O=C(/C=C/c1ccc(O)cc1)c1c(O)cc(O)cc1OCOC(O)CO</smiles><smiles>[R]c1cc(C(=O)/C=C/c2ccc(O)c(O)c2OC)ccc1O</smiles>

Licochalcone B (68) $\mathrm{R}=\mathrm{H}$

Tetrahydroxy-methoxychalcone (69) $\mathrm{R}=\mathrm{OH}$<smiles>CC(C)=CCc1cc(/C=C/C(=O)c2ccc(O)cc2O)cc(O)c1O</smiles>

Licoagrochalcone A (70)<smiles>COc1c(/C=C/C(=O)c2ccc(O)cc2)ccc(O)c1CC=C(C)C</smiles>

Licochalcone C (71)<smiles>C=CC(C)(C)c1cc(/C=C/C(=O)c2ccc(O)cc2O)c(OC)cc1O</smiles>

Licochalcone $\mathrm{G}$ (73)<smiles>CC(C)=CCCC1(C)C=Cc2c(O)c(CC=C(C)C)c(O)c(C(=O)/C=C/c3ccc(O)c(O)c3)c2O1</smiles>

Mallotophilippens E (76)<smiles>O=C(/C=C/c1ccc(O)cc1)c1c(O)cc(O)cc1O</smiles>

Figure 10. Cont. 
<smiles>[R]c1cc(/C=C/C(=O)c2ccc(O)c(C/C=C(\C)CC[C@H](O)C(=C)C)c2O)ccc1O</smiles>

2',3,4,4'-Tetrahydroxy-3'-(6-hydroxy-3,7-dimethyl-7-octadienyl)chalcone (79) $\mathrm{R}=\mathrm{OH}$ 2',4',4-Trihydroxy-3'-(6-hydroxy-3,7-dimethyl-7-octadienyl)chalcone (80) R = H<smiles>C=C(CCC=C(C)C)C(O)Cc1c(O)ccc(C(=O)/C=C/c2ccc(O)cc2)c1O</smiles>

2',4',4-Trihydroxy-3'-[2-hydroxy-7-methyl-3-methylene-6-octenyl-chalcone (81)<smiles>COc1ccc(/C=C/C(=O)c2ccccc2O)c(OC)c1OC</smiles>

2,3,4-Trimethoxy-2'-hydroxychalcone (82)<smiles>[R]c1cc(/C=C/C(=O)c2ccc([R20])c(C/C=C(/C)CCC=C(C)C)c2O)ccc1O</smiles>

Xanthoangelol (83) $\mathrm{R}=\mathrm{R}^{\prime}=\mathrm{H}$

Xanthoangel F (84) $\mathrm{R}=\mathrm{Me} ; \mathrm{R}^{\prime}=\mathrm{H}$

2',3,4,4'-Tetrahydroxy-3'-geranylchalcone (85) $\mathrm{R}=\mathrm{H}$; R'-<smiles>[R20]c1ccc(C(=O)/C=C/c2ccc(O)cc2)c(O)c1C/C=C(/C)CCC=C(C)C</smiles>

Xanthoangelol B (86) $\mathrm{R}=\mathrm{H}$

Xanthoangelol G (87) $\mathrm{R}=\mathrm{Me}$<smiles>COC(CC/C(C)=C/Cc1c(O)ccc(C(=O)/C=C/c2ccc(O)cc2)c1O)OC</smiles>

Xanthodeistal A (88)

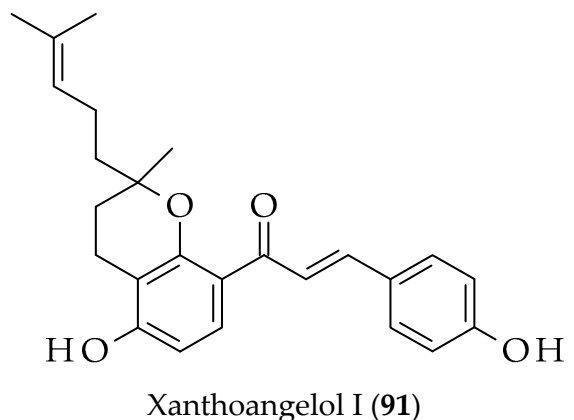

Figure 10. Structures of some naturally occurring bioactive chalcones.

Table 2. Bioactivities of some naturally occurring chalcones.

\begin{tabular}{llll}
\hline Bioactivities & Chalcones & Sources & References \\
\hline Anti-acetylcholinesterase & $\begin{array}{l}\text { Isosalipurposide (66) } \\
\text { Naringenin chalcone (78) }\end{array}$ & Acacia cyanophylla & [50] \\
\hline Anti-arthritic & Cardamonin (48) & Boesenbergia rotunda & [51] \\
\hline
\end{tabular}


Table 2. Cont.

\begin{tabular}{|c|c|c|c|}
\hline Bioactivities & Chalcones & Sources & References \\
\hline \multirow{30}{*}{ Anticancer } & \multirow{2}{*}{ Butein (16) } & Toxicodendron vernicifluum & [52] \\
\hline & & Butea dahlia & \multirow{2}{*}[22,53]{} \\
\hline & Calomelanone (17) & Stevia lucida & \\
\hline & Deoxydihydroxanthoangelol H (49) & \multirow{2}{*}{ Angelica keiskei } & \multirow{2}{*}[54]{} \\
\hline & 2,6-Dihydroxy-4-methoxychalcone (52) & & \\
\hline & Dimethyl-cardamonin (27) & Syzygium campanulatum & {$[55]$} \\
\hline & Echinantin (59) & Piper methysticum $\mathcal{E}$ Alpinia pricei & \multirow{3}{*}[56]{} \\
\hline & Flavokawain A (60) & \multirow{2}{*}{ Piper methysticum } & \\
\hline & Flavokawain B (28) & & \\
\hline & Flavokawain B (28) & Alpinia pricei & [57] \\
\hline & Flavokawain C (18) & Piper methysticum & \multirow{2}{*}[22,53]{} \\
\hline & Homobutein (19) & Butea frondosa & \\
\hline & $2^{\prime}$-Hydroxy-4', $5^{\prime}$-dimethoxychalcone (61) & Sarcandra hainanensis & [58] \\
\hline & 4-Hydroxychalcone (20) & \multirow{2}{*}{ Glycyrrhiza glabra } & \multirow{2}{*}[22,53]{} \\
\hline & 4'-Hydroxychalcone (21) & & \\
\hline & 4-Hydroxyderricin (62) & \multirow{2}{*}{ Angelica keiskei } & [59] \\
\hline & Isobavachalcone (63) & & [54] \\
\hline & Isobavachalcone (63) & Psoralea corylifolia & {$[60]$} \\
\hline & \multirow{3}{*}{ Isoliquiritigenin (22) } & Piper methysticum & \multirow{2}{*}{ [57] } \\
\hline & & Alpinia pricei & \\
\hline & & Glycyrrhiza glabra & {$[22,53]$} \\
\hline & Isosalipurposide (66) & Helichrysum maracandicum & {$[61]$} \\
\hline & Lonchocarpin (41) & Pongamia pinnata & {$[41]$} \\
\hline & Marein (77) & \multirow{2}{*}{ Glycyrrhiza glabra } & \multirow{2}{*}[22,53]{} \\
\hline & 4-Methoxychalcone (2) & & \\
\hline & Phloretin (23) & Stevia lucida & {$[22,53]$} \\
\hline & 2,3,4-Trimethoxy-2'-hydroxychalcone (82) & Piper methysticum & {$[62]$} \\
\hline & Xanthoangelol (83) & \multirow{3}{*}{ Angelica keiskei } & [59] \\
\hline & Xanthoangelol I (91) & & \multirow{2}{*}[54]{} \\
\hline & Xanthoangelol J (92) & & \\
\hline \multirow{3}{*}{ Antidiabetic } & $2^{\prime}, 6^{\prime}$-Dihydroxy-4'-methoxychalcone (54) & Piper claussenianum & [63] \\
\hline & 4-Hydroxyderricin (62) & A o li & $5<0$ \\
\hline & Xanthoangelol (83) & Angetil nets & {$[04]$} \\
\hline \multirow{8}{*}{ Anti-inflammatory } & Flavokawain B (28) & \multirow{2}{*}{ Alpinia pricei } & \multirow{2}{*}{ [65] } \\
\hline & Isoliquiritigenin (22) & & \\
\hline & Licoagrochalcone A (70) & \multirow{2}{*}{ Glycyrrhiza inflata } & \multirow{2}{*}[66]{} \\
\hline & Licochalcone B (68) & & \\
\hline & Licochalcone C (71) & Glycyrrhiza glabra & {$[67]$} \\
\hline & Mallotophilippens C (74) & \multirow{3}{*}{ Mallotus philippinensis } & \multirow{3}{*}[68]{} \\
\hline & Mallotophilippens D (75) & & \\
\hline & Mallotophilippens E (76) & & \\
\hline
\end{tabular}


Table 2. Cont

\begin{tabular}{|c|c|c|c|c|}
\hline Bioactivities & & Chalcones & Sources & References \\
\hline \multicolumn{5}{|l|}{ Antimicrobial } \\
\hline & \multirow{5}{*}{ Antibacterial } & $2^{\prime}, 4^{\prime}$-Dihydroxychalcone (51) & Zuccagnia punctata & [69] \\
\hline & & $\begin{array}{l}3,2^{\prime} \text {-Dihydroxy-2,4,4' } 6^{\prime}- \\
\text { tetramethoxychalcone }(55)\end{array}$ & \multirow{3}{*}{ Piper hispidum } & \multirow{3}{*}[70]{} \\
\hline & & $\begin{array}{l}2^{\prime} \text {-Hydroxy- } 2,4,4^{\prime}, 6^{\prime}- \\
\text { tetramethoxychalcone }(56)\end{array}$ & & \\
\hline & & $\begin{array}{l}2^{\prime} \text {-Hydroxy-2,3,4,4', } 6^{\prime}- \\
\text { pentamethoxychalcone (57) }\end{array}$ & & \\
\hline & & Isoliquiritigenin (22) & Apis mellifera & {$[71]$} \\
\hline & \multirow{13}{*}{ Antifungal } & $\begin{array}{l}\text { 1-(5,7-Dihydroxy-2,2,6-trimethyl-2H-1- } \\
\text { benzopyran-8-yl)-3- } \\
\text { phenyl-2-propen-1-one [58] }\end{array}$ & Mallotus philippinensis & {$[72]$} \\
\hline & & $\begin{array}{l}3,2^{\prime} \text {-Dihydroxy-2,4,4' } 6^{\prime}- \\
\text { tetramethoxychalcone }(55)\end{array}$ & \multirow{3}{*}{ Piper hispidum } & \multirow{3}{*}[70]{} \\
\hline & & $\begin{array}{l}2^{\prime} \text {-Hydroxy-2,4,4' } 4^{\prime}, 6^{\prime}- \\
\text { tetramethoxychalcone (56) }\end{array}$ & & \\
\hline & & $\begin{array}{l}2^{\prime} \text {-Hydroxy-2,3,4,4', } 6^{\prime}- \\
\text { pentamethoxychalcone (57) }\end{array}$ & & \\
\hline & & $4^{\prime}$-Hydroxyrottlerin $(64)$ & Mallotus philippinensis & [72] \\
\hline & & Isoliquiritigenin (22) & Apis mellifera & {$[71]$} \\
\hline & & Kamalachalcone E (67) & \multirow{2}{*}{ Mallotus philippinensis } & \multirow{2}{*}{ [72] } \\
\hline & & Rottlerin (65) & & \\
\hline & & $\begin{array}{l}2^{\prime}, 3,4,4^{\prime} \text {-Tetrahydroxy-3' } \\
\text { geranylchalcone }(85)\end{array}$ & \multirow{5}{*}{ Artocarpus nobilis } & \multirow{5}{*}{ [73] } \\
\hline & & $\begin{array}{l}\text { 2',3,4,4'-Tetrahydroxy-3'-(6-hydroxy-3,7- } \\
\text { dimethyl-7-octadienyl)chalcone (79) }\end{array}$ & & \\
\hline & & $\begin{array}{l}2^{\prime}, 4^{\prime}, 4 \text {-Trihydroxy-3'-geranylchalcone (83) } \\
\text { [xanthoangelol] }\end{array}$ & & \\
\hline & & $\begin{array}{l}2^{\prime}, 4^{\prime}, 4 \text {-Trihydroxy-3'-(6-hydroxy-3,7- } \\
\text { dimethyl-7-octadienyl)chalcone }(\mathbf{8 0})\end{array}$ & & \\
\hline & & $\begin{array}{l}2^{\prime}, 4^{\prime}, 4 \text {-Trihydroxy-3'-[2-hydroxy-7- } \\
\text { methyl-3- } \\
\text { methylene-6-octenyl-chalcone] (81) }\end{array}$ & & \\
\hline & \multirow{13}{*}{ Antiviral } & Dihydrochalcone diglycoside (50) & Thalassodendrin ciliatum & [74] \\
\hline & & Echinantin (59) & \multirow{2}{*}{ Glycyrrhiza inflata } & \multirow{2}{*}{ [75] } \\
\hline & & Isoliquiritigenin (22) & & \\
\hline & & \multirow{2}{*}{ Licochalcone A (40) } & Quercus coccifera & [76] \\
\hline & & & Glycyrrhiza inflata & [75] \\
\hline & & Licochalcone B (68) & Quercus coccifera & [76] \\
\hline & & Licochalcone D (72) & \multirow{3}{*}{$\begin{array}{l}\text { Glycyrrhiza inflata } \\
\text { Quercus coccifera }\end{array}$} & \multirow{2}{*}[76]{} \\
\hline & & Licochalcone G (73) & & \\
\hline & & Tetrahydroxy-methoxychalcone (69) & & [76] \\
\hline & & Xanthoangelol (83) & \multirow{4}{*}{ Angelica keiskei } & \multirow{4}{*}{ [77] } \\
\hline & & Xanthoangelol F (84) & & \\
\hline & & Xanthoangelol D (89) & & \\
\hline & & Xanthoangelol E (90) & & \\
\hline
\end{tabular}


Table 2. Cont

\begin{tabular}{|c|c|c|c|}
\hline Bioactivities & Chalcones & Sources & References \\
\hline & Xanthoangelol B (86) & & \\
\hline & Xanthoangelol G (87) & & \\
\hline & Xanthodeistal A (88) & & \\
\hline \multirow[b]{2}{*}{ Antioxidant } & Licochalcone C (71) & Glycyrrhiza glabra & [67] \\
\hline & $\begin{array}{l}\text { Isosalipurposide (66) } \\
\text { Naringenin chalcone (78) }\end{array}$ & Acacia cyanophylla & {$[50]$} \\
\hline \multirow{4}{*}{ Antiparasitic } & $\begin{array}{l}\text { (E)-1-(2,4-Dihydroxy-3-(3-methylbut-2- } \\
\text { en-1-yl)phenyl)-3- } \\
\text { phenylprop-2-en-1-one (53) }\end{array}$ & Lonchocarpus sp. & [78] \\
\hline & Flavokawain B (28) & Polygonum ferrugineum & [79] \\
\hline & Licochalcone C (71) & Glycyrrhiza glabra & [80] \\
\hline & Licoagrochalcone A (70) & Erythrina abyssinica & [81] \\
\hline \multirow{3}{*}{ Immunoregulatory } & Mallotophilippens C (74) & \multirow{3}{*}{ Mallotus philippinensis } & \multirow{3}{*}{ [68] } \\
\hline & Mallotophilippens D (75) & & \\
\hline & Mallotophilippens E (76) & & \\
\hline
\end{tabular}

\subsection{Anticancer and Cancer Chemopreventive Activity}

Several investigations have revealed the bioactivity of chalcones against cancer, either as a cancer chemopreventive agent or as a potential cancer curative agent. However, most of those investigations were in vitro or in silico. Both natural (Figure 10; Table 2) and synthetic chalcones acted as anticancer agents through the mechanisms sated in Table 1 , and mainly the induction of cancer/tumor cell death, predominantly by apoptosis. Research groups who are interested in the cytotoxicity of chalcones, such as Sumiyoshi et al. [59], reported that two chalcone derivatives, xanthoangelol (83) and 4-hydroxyderricin (62), isolated from Angelica keiskei roots could inhibit metastasis and the growth of a tumor. In addition, twenty-one natural chalcones were investigated by Orlikova et al. [22] for their ability to inhibit NF-KB in K562 cells. They found that butein (16), calomelanone (17), flavokawain C (18), homobutein (19), 4-hydroxychalcone (20), 4'-hydroxychalcone (21), isoliquiritigenin (22), marein (77), 4-methoxychalcone (2), and phloretin (23) had the ability to inhibit histone deacetylase enzyme (HDAC), and NF- $\mathrm{kB}$.

Inspired by the anticancer potential of naturally occurring chalcones, several synthetic chalcone analogs were produced and assessed for their anticancer properties. For example, Sakagami et al. [82] reported the synthesis of fifteen chalcones (Figure 11), and showed their ability to act as selective anticancer agents in several human oral squamous cancer cell lines, such as HSC-2, HSC-3, HSC-4, and Ca9-22.

Anticancer activity and possible mechanisms of action of $2^{\prime}$-hydroxy-4 $4^{\prime}, 5^{\prime}$-dimethoxychalcone (61), isolated from Sarcandra hainanensis, were investigated using the lung cancer cell lines, e.g., H322M, H460, H358, H1792 and H157. It was shown that chalcone 61 could increase the level of cellular reactive oxygen species (ROS) and induce cell apoptosis. Ramirez-Tagle et al. [62] used 3'-bromo-3,4-dimethoxychalcone (107) and 2,3,4-trimethoxy2'-hydroxychalcone (82) on mouse hepatocytes (HepM) and human hepatoma cells (HepG2 and Huh-7) and found that both chalcones could lead to cellular apoptosis and to an increase ROS levels. Synthetic chalcone (E)-1-(2-hydroxyphenyl)-3-(4-methyl-phenyl)-prop2-en-1-one (108) was identified as a chemopreventive agent with the results indicating that it could decrease the extent of DNA damage [83]. 
<smiles>[R]c1ccc(/C=C/C(=O)c2ccccc2O)cc1[R]</smiles>

2',4-Dihydroxychalcone (93) $\mathrm{R}=\mathrm{H} ; \mathrm{R}^{\prime}=\mathrm{OH}$ 4-Bromo-2'-hydroxychalcone (94) $\mathrm{R}=\mathrm{H} ; \mathrm{R}^{\prime}=\mathrm{Br}$ 4-Chloro-2'-hydroxychalcone (95) $\mathrm{R}=\mathrm{H} ; \mathrm{R}^{\prime}=\mathrm{Cl}$

4-Fluoro-2'-hydroxychalcone (96) $\mathrm{R}=\mathrm{H} ; \mathrm{R}^{\prime}=\mathrm{F}$ 2'-Hydroxychalcone (6) $\mathrm{R}=\mathrm{R}^{\prime}=\mathrm{H}$

2'-Hydroxy-4-methoxychalcone (97) $\mathrm{R}=\mathrm{H}$; $\mathrm{R}^{\prime}=\mathrm{OMe}$ 2'-Hydroxy-3,4-dimethoxychalcone (98) $\mathrm{R}=\mathrm{R}^{\prime}=\mathrm{OMe}$<smiles>[X]c1ccc(/C=C/C(=O)c2ccc(OC)cc2O)cc1[R]</smiles>

4-Bromo-2'-hydroxy-4'-methoxychalcone (99) $\mathrm{R}=\mathrm{H} ; \mathrm{R}^{\prime}=\mathrm{Br}$

2',4-Dihydroxy-4'methoxychalcone (100) $\mathrm{R}=\mathrm{H} ; \mathrm{R}^{\prime}=\mathrm{OH}$

2'-Hydroxy-4,4'-dimethoxyflavone (101) $\mathrm{R}=\mathrm{H} ; \mathrm{R}^{\prime}=\mathrm{OMe}$

2'-Hydroxy-4'-methoxychalcone (102) $\mathrm{R}=\mathrm{R}^{\prime}=\mathrm{H}$

2'-Hydroxy-3,4,4'-trimethoxyflavone (103) $R=R^{\prime}=\mathrm{OMe}$<smiles>[R]c1cc(OC)ccc1/C=C/C(=O)c1ccc(OC)cc1[R]</smiles>

4,4'-Dimethoxychalcone (104) $\mathrm{R}=\mathrm{R}^{\prime}=\mathrm{H}$

2,4,4'-Trimethoxychalcone (105) $\mathrm{R}=\mathrm{H} ; \mathrm{R}^{\prime}=\mathrm{OMe}$

2',4,4'-Trimethoxychalcone (106) $\mathrm{R}=\mathrm{OMe} ; \mathrm{R}^{\prime}=\mathrm{H}$<smiles>COc1ccc(/C=C/C(=O)c2cccc(Br)c2)cc1OC</smiles>

3'-Bromo-3,4-dimethoxychalcone (107)<smiles>Cc1ccc(/C=C/C(=O)c2ccccc2O)cc1</smiles>

(E)-1-(2-Hydroxyphenyl)-3-(4-methyl-phenyl)prop-2-en-1-one) (108)

Figure 11. Structures of some synthetic chalcones with anticancer potential.

\subsection{Antidiabetic Activity}

Some naturally occurring chalcones were shown to possess antidiabetic properties. For example, 2' $6^{\prime}$-dihydroxy-4'-methoxychalcone (52), isolated from the flowers of Piper claussenianum [63], and 4-hydroxyderricin (62), and xanthoangelol (83) from Angelica keiskei, were found to have antidiabetic properties [64]. Chalcone 52 exhibited its antidiabetic properties through producing a hypoglycemic effect in streptozotocin-induced diabetes in rats. It was observed that this compound could reduce blood glucose levels from $277.4 \mathrm{mg} / \mathrm{dL}$ before treatment to $158.8 \mathrm{mg} / \mathrm{dL}$ after 12 days of treatment. Both 4-hydroxyderricin (62) and xanthoangelol (83) displayed insulin-like behavior via a pathway that is not dependent on the activation of peroxisome proliferator-activated receptor- $\gamma$ [64], whereas chalcone 62 could prevent the progression of diabetes in genetically diabetic $\mathrm{KK}-\mathrm{A}^{\mathrm{y}}$ mice.

The antidiabetic effect of naturally occurring chalcones prompted several bioinspired syntheses of chalcone analogs, which were subsequently tested for their antidiabetic poten- 
tial. For instance, synthetic chalcones, (E)-1,3-dip-tolylprop-2-en-1-one (114), 4,5-dihydro-3,5dip-tolylpyrazole-1-carbothioamide (110), 4,5-dihydro-3,5-dip-tolylpyrazole-1-carboxamide (111), (4,5-dihydro-3,5-dip-tolylpyrazol-1-yl)(phenyl)methanone (112), 4,5-dihydro-1-phenyl3,5-dip-tolyl-1 $H$-pyrazole (113), and 3,5-bis(4-methylphenyl)-1-(phenylsulfonyl)-4,5-dihydro$1 H$-pyrazole (109), were shown to act as hypoglycemic agents by decreasing the level of blood glucose in alloxan-induced diabetic rats [84]. Among these chalcone derivatives, 4,5-dihydro-1-phenyl-3,5-dip-tolyl- $1 \mathrm{H}$-pyrazole (113) produced the most prominent antidiabetic effect. In addition, Chinthala et al. [85] synthesized twenty chalcone derivatives, three of which (115-117) (Figure 12) were found to exert $\alpha$-glucosidase-inhibitory activity $\left(\mathrm{IC}_{50}=67.7-102.1 \mu \mathrm{M}\right)$. Based on the obtained results with these chalcone derivatives, it was assumed that the presence of aliphatic chain length up to five carbons (n-pentyl) attached to the triazole ring might be optimum for generating $\alpha$-glucosidase activity.

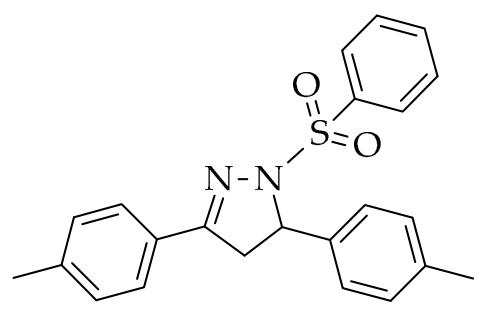

3,5-bis(4-Methylphenyl)-1-(phenylsulfonyl)4,5-dihydro-1H-pyrazole (109)<smiles>Cc1ccc(C2=NN(C(N)=O)C(c3ccc(C)cc3)C2)cc1</smiles>

4,5-Dihydro-3,5-dip-tolylpyrazole-1-carboxamide (111)<smiles>Cc1ccc(C2=NN(c3ccccc3)C(c3ccc(C)cc3)C2)cc1</smiles><smiles>Cc1ccc(C2=NN(C(N)S)C(c3ccc(C)cc3)C2)cc1</smiles>

4,5-Dihydro-3,5-dip-tolylpyrazole-1-carbothioamide (110)<smiles>Cc1ccc(C2=NN(C(=O)c3ccccc3)C(c3ccc(C)cc3)C2)cc1</smiles>

(4,5-Dihydro-3,5-dip-tolylpyrazol-1-yl)(phenyl)methanone (112)<smiles>Cc1ccc(/C=C/C(=O)c2ccc(C)cc2)cc1</smiles>

(E)-1,3-Dip-tolylprop-2-en-1-one (114)

4,5-Dihydro-1-phenyl-3,5-dip-tolyl-1H-pyrazole (113)<smiles>[R]c1cc2c(c([R])c1C(=O)/C=C/c1ccc(OCc3cn([R])nn3)cc1)CCC(C)(C)O2</smiles>

Chalcone 1,2,3-triazole derivatives

Chalcone-triazole 1 (115) $\mathrm{R}=2 \mathrm{C}_{2} \mathrm{H}_{5}$-phenyl; $\mathrm{R}^{\prime}=\mathrm{H} ; \mathrm{R}^{\prime \prime}=\mathrm{OH}$

Chalcone-triazole 2 (116) $\mathrm{R}=n$-pentyl; $\mathrm{R}^{\prime}=\mathrm{H} ; \mathrm{R}^{\prime \prime}=\mathrm{OH}$

Chalcone-triazole 3 (117) $\mathrm{R}=n$-pentyl; $\mathrm{R}^{\prime}=\mathrm{OH} ; \mathrm{R}^{\prime \prime}=\mathrm{H}$

Figure 12. Structures of some synthetic antidiabetic chalcones. 


\subsection{Anti-Inflammatory Activity}

Naturally occurring chalcones are phenolic compounds, and often possess one or more phenolic hydroxyl functionality in their structures, which generally offer them with the inherent free-radical-scavenging properties that can be useful against oxidative stress. It is known that oxidative stress is associated with inflammatory responses. Thus, any reduction in oxidative stress is expected to inhibit inflammatory responses. Chalcones with free-radical-scavenging properties were shown to also possess anti-inflammatory properties. The examples of naturally occurring anti-inflammatory chalcones could include flavokawain B (28) and isoliquirigenin (22) from Alpinia pricei [65], licoagrochalcone A (70) and licochalcone B (68) from Glycyrrhiza inflata [66], licochalcone C (71) from Glycyrrhiza glabra [67], and mallotophilippens C-E (74-76) from Mallotus philippinensis [68]. It was shown that flavokawain B (28) could inhibit the production of nitric oxide (NO) and prostaglandin $\mathrm{E}_{2}$ (PGE2) in lipopolysaccharide (LPS)-induced murine leukemia macrophage RAW 264.7 cells [65]. Furthermore, this chalone dose-dependently decreased the secretion of TNF-R and inhibited the expression of iNOS (inducible nitric oxide synthase) and COX2 (cyclooxygenase 2) proteins. Flavokawain B (28) was also found to block the nuclear translocation NF- $\mathrm{kB}$ and thus decrease NF- $\mathrm{kB}$ protein levels in the nucleus. Similar anti-inflammatory activity was observed with the chalcone $\mathbf{2 8}$ in vivo using a mouse model, where the pre-administration of a $200 \mathrm{mg} / \mathrm{kg}$ compound could reduce the NO concentration and significantly suppress LPS-induced iNOS, COX-2, and NF- $k B$ proteins expression in mouse liver. Licoagrochalcone A (70) and licochalcone $B(68)$ showed prominent anti-inflammatory activity with $\mathrm{IC}_{50}$ values of 9.35 and $8.78 \mu \mathrm{M}$, respectively, on LPS-induced NO production. Additionally, chalcone $\mathbf{7 0}$ showed potent inhibitory effect on NF- $\mathrm{BB}$ transcription [66]. Mallotophilippens C-E (74-76) were found to inhibit NO production in activated RAW 264.7 cells and the expression of iNOS, COX2, IL-6 (interleukin 6), and IL-1 $\beta$ mRNA [68], suggesting that these compounds might exert their anti-inflammatory properties through inactivation of NF- $\mathrm{KB}$.

Anti-inflammatory properties of naturally occurring chalcones, as exemplified above, prompted bioinspired synthesis of several chalcone analogs as anti-inflammatory agents [86-89]. Over two decades ago, several 2'-hydroxychalcones and 2',5'-dihydroxychalcones (Figure 13) were synthesized and their anti-inflammatory properties were ascertained by observation of their in vitro inhibitory effects on the activation of mast cells, neutrophils, microglial cells, and macrophages [86]. Among these synthetic chalcones, 2,2'-hydroxychalcone (124) emerged as the most potent anti-inflammatory agent that could inhibit the release of $\beta$ glucuronidase $\left(\mathrm{IC}_{50}=1.6 \mu \mathrm{M}\right)$ and lysozyme $\left(\mathrm{IC}_{50}=1.4 \mu \mathrm{M}\right)$ from rat neutrophils. Later, eleven chalcone derivatives (Figure 13) were synthesized by the Claisen-Schmidt condensation of acetophenones and aromatic aldehydes, or produced with suitable dihydrochalcone and alkyl bromide, or prepared in one-pot synthesis involving acetophenone and aromatic aldehyde under ultrasonication [87], and their anti-inflammatory potential was assessed. Chalcone derivatives 125, 132, 133, and 136 showed a considerable level of inhibition on the release of $\beta$-glucuronidase or lysozyme from rat neutrophils stimulated with appropriate stimuli; chalcones $\mathbf{1 2 5}$ and $\mathbf{1 3 3}$ inhibited superoxide anion production in rat neutrophils; and chalcones 123, 128, and 135 could inhibit NO production. Jantan et al. [88] reported the synthesis of chalcone derivatives and showed their potential as inhibitors of secretory phospholipase A2, cyclooxygenases, lipoxygenase, and pro-inflammatory cytokines by a series of in vitro enzyme inhibition and in silico assays. Chalcone derivatives with 4-methylamino-ethanol functionality appeared to be the most potent ones. Most recently, a synthetic chalcone (139) (Figure 13) was demonstrated to possess antioxidant, anti-inflammatory, and neuroprotective properties [89]. This chalcone could attenuate LPS-induced inflammation in RAW 264.7 macrophages. 
<smiles>[R]c1ccc([R])c(C(=O)/C=C/c2ccc([Y])c([R7])c2[R])c1</smiles>

4-Chloro-2'-hydroxychalcone (118) $\mathrm{R}=\mathrm{R}^{\prime \prime}=\mathrm{R}^{\prime \prime \prime}=\mathrm{H} ; \mathrm{R}^{\prime}=\mathrm{OH} ; \mathrm{R}^{\prime \prime \prime \prime}=\mathrm{Cl}$

4-Chloro-2'5'-dimethoxychalcone (119) $\mathrm{R}=\mathrm{R}^{\prime}=\mathrm{OMe} ; \mathrm{R}^{\prime \prime}=\mathrm{R}^{\prime \prime \prime}=\mathrm{H} ; \mathrm{R}^{\prime \prime \prime \prime}=\mathrm{Cl}$

4-Chloro-2'5'-diethoxychalcone (120) $\mathrm{R}=\mathrm{R}^{\prime}=\mathrm{OEt}$; $\mathrm{R}^{\prime \prime}=\mathrm{R}^{\prime \prime \prime}=\mathrm{H} ; \mathrm{R}^{\prime \prime \prime \prime}=\mathrm{Cl}$

4-Chloro-2'5'-dipropyloxychalcone (121) $\mathrm{R}=\mathrm{R}^{\prime}=\mathrm{OPr}$; $\mathrm{R}^{\prime \prime}=\mathrm{R}^{\prime \prime \prime}=\mathrm{H}$; $\mathrm{R}^{\prime \prime \prime \prime}=\mathrm{Cl}$

4-Chloro-2'5'-dibutyloxychalcone (122) $\mathrm{R}=\mathrm{R}^{\prime}=\mathrm{OBu} ; \mathrm{R}^{\prime \prime}=\mathrm{R}^{\prime \prime \prime}=\mathrm{H} ; \mathrm{R}^{\prime \prime \prime \prime}=\mathrm{Cl}$

3,4-Dichloro-2'-hydroxychalcone (123) $\mathrm{R}=\mathrm{R}^{\prime \prime}=\mathrm{H} ; \mathrm{R}^{\prime}=\mathrm{OH} ; \mathrm{R}^{\prime \prime \prime}=\mathrm{R}^{\prime \prime \prime \prime}=\mathrm{Cl}$

2,2'-Dihydroxychalcone (124) $\mathrm{R}=\mathrm{R}^{\prime \prime \prime}=\mathrm{R}^{\prime \prime \prime \prime}=\mathrm{H} ; \mathrm{R}^{\prime}=\mathrm{R}^{\prime \prime}=\mathrm{OH}$

$2^{\prime}$,4-Dihydroxychalcone (125) $\mathrm{R}=\mathrm{R}^{\prime \prime}=\mathrm{R}^{\prime \prime \prime}=\mathrm{H} ; \mathrm{R}^{\prime}=\mathrm{R}^{\prime \prime \prime \prime}=\mathrm{OH}$

4,5'-Dimethoxy-2'-hydroxychalcone (126) $\mathrm{R}=\mathrm{R}^{\prime \prime \prime}=\mathrm{OMe} ; \mathrm{R}^{\prime}=\mathrm{OH} ; \mathrm{R}^{\prime \prime}=\mathrm{R}^{\prime \prime \prime}=\mathrm{H}$

3,4-Dihydroxy-2', $5^{\prime}$-dimethoxychalcone (127) $\mathrm{R}=\mathrm{R}^{\prime}=\mathrm{OMe}: \mathrm{R}^{\prime \prime}=\mathrm{H} ; \mathrm{R}^{\prime \prime \prime}=\mathrm{R}^{\prime \prime \prime \prime}=\mathrm{OH}$

4-Hydroxy-2', $5^{\prime}$-dimethoxychalcone (128) $\mathrm{R}=\mathrm{R}^{\prime}=\mathrm{OMe} ; \mathrm{R}^{\prime \prime}=\mathrm{R}^{\prime \prime \prime}=\mathrm{H} ; \mathrm{R}^{\prime \prime \prime \prime}=\mathrm{OH}$

2'-Hydroxy-4-chloro-5'-methoxychalcone (129) $\mathrm{R}=\mathrm{OMe} ; \mathrm{R}^{\prime}=\mathrm{OH} ; \mathrm{R}^{\prime \prime}=\mathrm{R}^{\prime \prime \prime}=\mathrm{H} ; \mathrm{R}^{\prime \prime \prime \prime}=\mathrm{Cl}$

2'-Hydroxy-4-methy-5'-methoxychalcone (130) $\mathrm{R}=\mathrm{OMe} ; \mathrm{R}^{\prime}=\mathrm{OH} ; \mathrm{R}^{\prime \prime}=\mathrm{R}^{\prime \prime \prime}=\mathrm{H} ; \mathrm{R}^{\prime \prime \prime \prime}=\mathrm{Me}$

3,4,5'-Trimethoxy-2'-hydroxychalcone (131) $\mathrm{R}=\mathrm{R}^{\prime \prime \prime}=\mathrm{R}^{\prime \prime \prime}=\mathrm{OMe} ; \mathrm{R}^{\prime}=\mathrm{OH} ; \mathrm{R}^{\prime \prime}=\mathrm{H}$<smiles>O=C(/C=C/c1cccs1)c1ccccc1O</smiles>

2'-Hydroxy-thiochalcone 1 (132)<smiles>O=C(/C=C/C1=CCCS1)c1ccccc1O</smiles>

2'-Hydroxy-thiochalcone 2 (133)<smiles>O=C(/C=C/c1ccc(O)c(O)c1)c1cccs1</smiles>

Thiochalcone (134)<smiles>CC(C)(C)c1cc(/C=C/C(=O)c2cc(O)ccc2O)cc(C(C)(C)C)c1O</smiles>

2',5',4-Trihydroxy-3,5-di-isopropyl-chalcone (135)<smiles>[R]c1ccc(/C=C/C(=O)c2cc(O)ccc2O)cc1</smiles>

4-Chloro-2',5'-dihydroxychalcone (137) $\mathrm{R}=\mathrm{Cl}$ 2',5'-Dihydroxychalcone $(\mathbf{1 3 8}) \mathrm{R}=\mathrm{H}$<smiles>O=C(/C=C/c1c[nH]c2ccccc12)c1cc(O)ccc1O</smiles>

Nitrogen ring containing chalcone (136)<smiles>COc1ccc(/C=C/C(=O)c2ccccc2O)cc1</smiles>

2'-Hydroxy-4-methoxychalcone (139)

Figure 13. Structures of some synthetic anti-inflammatory chalcones.

\subsection{Antimicrobial Activity}

There are several reports available in the literature describing the antimicrobial activity of natural and synthetic chalcones; these activities include their activity against several pathogenic bacterial, fungal, and viral species. Figure 10 and Table 2 present some examples 
of naturally occurring antimicrobial chalcones. Here, the antimicrobial activity of chalcones is discussed under three subsections: antibacterial, antifungal, and antiviral chalcones.

\subsubsection{Antibacterial Activity}

Antibacterial activity of naturally occurring chalcones is well-documented in the literature $[69,70]$ (Table 2). For example, $2^{\prime}, 4^{\prime}$-dihydroxychalcone (51) isolated from Zuccagnia punctata; 3,2'-dihydroxy-2,4,4', $6^{\prime}$-tetramethoxychalcone (55), 2'-hydroxy-2,4,4',6' -tetramethoxychalcone (56), and 2'-hydroxy-2,3,4,4',6'-pentamethoxychalcone (57) from Piper hispidum; and isoliquiritigenin (22) from the Brazilian propolis (Apis melifera) were shown to possess antibacterial properties [69-71]. 2', $4^{\prime}$-Dihydroxychalcone (51) was found active against the Gram-positive and Gram-negative bacterial species, including Acinetobacter baumannii, Enterobacter cloacae, Morganella morganii, Proteus mirabilis, Pseudomonas aeruginosa, Serratia marcescens, and Stenotrophomonas maltophilia, which have MIC values ranging between $0.10 \mu \mathrm{g} / \mathrm{mL}$ and $100 \mu \mathrm{g} / \mathrm{mL}$ [69]. 3,2'-Dihydroxy-2,4,4',6'-tetramethoxychalcone (55), 2'-hydroxy-2,4,4', $6^{\prime}$ tetramethoxychalcone (56) and $2^{\prime}$-hydroxy-2,3,4,4',6 $6^{\prime}$-pentamethoxychalcone (57) were shown active against Staphylococcus aureus with MIC values in the range of $125-250 \mu \mathrm{g} / \mathrm{mL}$ [70], whilst the antibacterial activity isoliquiritigenin (22) was observed against Actinomyces naeslundii, Staphylococcus aureus, and Streptococcus mutans (MIC $=15.6-62.5 \mu \mathrm{g} / \mathrm{mL}$ ) [71].

The antibacterial activity of natural chalcones inspired synthetic chemists to attempt bioinspired synthesis of antibacterial chalcone derivatives. For example, a decade ago, Solankee et al. [90] investigated synthetic triazine-based chalcones (Figure 14) as antibacterial agents, and reported inhibition of the growth of Bacillus cereus, Enterobacter cloacae, Escherichia coli, Listeria monocytogenes, Micrococcus flavus, Pseudomonas aeruginosa, Salmonella typhimurium, and Staphylococcus aureus. However, except for the furanyl derivative (143), the activity of other compounds was rather weak. Chu et al. [91] resynthesized 29 cationic chalcones with varying aryl substitutions, and diversity in the $N, N$-dimethyl alkylamine length, and tested all compounds against Enterococcus faecalis, Escherichia coli, Salmonella enterica and Staphylococcus aureus. Chalcone derivatives, (E)-N-(2-((4-cinnamoylphenyl)amino)-2-oxoethyl)- $N, N$-dimethyloctan-1-aminium chloride (145) and (E)-N-(2-((4-(3-(2-fluorophenyl)acryloyl)phenyl)amino)-2-oxoethyl)- $N, N$ dimethyloctan-1-aminium chloride (146) (Figure 14), demonstrated a considerable level of growth inhibitory activity against both Gram-negative and Gram-positive bacteria, including the drug-resistant New Delhi metallo- $\beta$-lactamase-1 (NDM), Klebsiella pneumoniae carbapenemase (KPC), and methicillin-resistant Staphylococcus aureus (MRSA) strains. While (E)-N-(2-((4-cinnamoylphenyl)amino)-2-oxoethyl)- $N, N$-dimethyloctan-1-aminium chloride (145) was most active against $E$. coli $(\mathrm{MIC}=2 \mu \mathrm{g} / \mathrm{mL})$, derivative 146 showed the most prominent activity against $S$. aureus $(\mathrm{MIC}=0.5 \mu \mathrm{g} / \mathrm{mL}$ ). This finding demonstrated the potential applications of chalcone peptidomimetics as a new class of antibacterial agents.

Most recently, a series of $\beta$-chalcone derivatives (147-158) (Figure 14) was synthesized using various substituted amines by the Claisen-Schmidt condensation reaction in basic condition, and evaluated for antibacterial activity against different species of bacteria, including Enterococcus faecalis, Escherichia coli, Klebsiella pneumoniae, Pseudomonas aeruginosa, Salmonella enterica and Staphylococcus pneumoniae [92]. Among these synthetic chalcone derivatives, compound $\mathbf{1 4 8}$ emerged as the most potent antibacterial agent which was particularly effective against $E$. coli $(\mathrm{MIC}=125 \mu \mathrm{g} / \mathrm{mL})$. In silico studies established DNA binding and molecular docking of these compounds. There are several other examples of synthesis antibacterial chalcones and assessment of their modes of action available in the literature [93-97]. 
<smiles>[R]c1ccc(/C=C/C(=O)c2ccc(Nc3nc(Nc4ccccc4)nc(Nc4ccccc4)n3)cc2)c([R])c1</smiles>

Triazine chalcone 1 (140) $\mathrm{R}=\mathrm{NO}_{2} ; \mathrm{R}^{\prime}=\mathrm{H}$

Triazine chalcone $2(\mathbf{1 4 1}) \mathrm{R}=\mathrm{Cl} ; \mathrm{R}^{\prime}=\mathrm{H}$

Triazine chalcone 3 (142) $\mathrm{R}=\mathrm{H} ; \mathrm{R}^{\prime}=\mathrm{OMe}$<smiles>[R]C=CC(=O)c1ccc(Nc2nc(Nc3ccccc3)nc(Nc3ccccc3)n2)cc1</smiles>

Triazine chalcone 4 (143) R = Furanyl Triazine chalcone 5 (144) $\mathrm{R}$ = Thienyl<smiles>[R]c1ccccc1/C=C/C(=O)c1ccc(NC(=O)C[N+](C)(C)Cl)cc1</smiles>

(E)-N-(2-((4-cinnamoylphenyl)amino)-2-oxoethyl)- $N, N$-dimethyloctan-1-aminium chloride (145) $\mathrm{R}=\mathrm{H}$ (E)-N-(2-((4-(3-(2-Fluorophenyl)acryloyl)phenyl)amino)-2-oxoethyl)-N,N-dimethyloctan-1-aminium chloride (146) $\mathrm{R}=\mathrm{F}$<smiles>[R]c1ccc(C(=O)/C=C/c2c[nH]c3ccccc23)cc1[R]</smiles><smiles>[R]c1cccc(C(=O)/C=C/c2ccccn2)c1</smiles>

$\beta$-Chalcone derivative $\mathrm{d}(\mathbf{1 5 0}) \mathrm{R}=\mathrm{NO}_{2}$ $\beta$-Chalcone derivative e (151) $\mathrm{R}=\mathrm{Br}$

$\beta$-Chalcone derivative a (147) $\mathrm{R}=\mathrm{NO}_{2} ; \mathrm{R}^{\prime}=\mathrm{H}$

$\beta$-Chalcone derivative $b(\mathbf{1 4 8}) \mathrm{R}=\mathrm{H} ; \mathrm{R}^{\prime}=\mathrm{Me}$

$\beta$-Chalcone derivative c (149) $\mathrm{R}=\mathrm{H} ; \mathrm{R}^{\prime}=\mathrm{NO}_{2}$

Figure 14. Cont. 
<smiles>[R]c1ccc(C(=O)/C=C/c2c[nH]cn2)cc1[R]</smiles>

$\beta$-Chalcone derivative $\mathrm{f}(\mathbf{1 5 2}) \mathrm{R}=\mathrm{NO}_{2} ; \mathrm{R}^{\prime}=\mathrm{H}$ $\beta$-Chalcone derivative $g(\mathbf{1 5 3}) \mathrm{R}=\mathrm{H} ; \mathrm{R}^{\prime}=\mathrm{Me}$ $\beta$-Chalcone derivative $h(\mathbf{1 5 4}) \mathrm{R}=\mathrm{Br} ; \mathrm{R}^{\prime}=\mathrm{H}$<smiles>[R]c1ccc(C(=O)/C=C/c2c(O)ccc3ccccc23)cc1[R]</smiles>

$\beta$-Chalcone derivative i (155) $\mathrm{R}=\mathrm{NO}_{2} ; \mathrm{R}^{\prime}=\mathrm{H}$

$\beta$-Chalcone derivative $\mathrm{j}(\mathbf{1 5 6}) \mathrm{R}=\mathrm{H} ; \mathrm{R}^{\prime}=\mathrm{NO}_{2}$

$\beta$-Chalcone derivative $\mathrm{k}\left(\right.$ (157) $\mathrm{R}=\mathrm{Br} ; \mathrm{R}^{\prime}=\mathrm{H}$<smiles>O=C(/C=C/c1ccc(O)c(O)c1)c1cccc([N+](=O)[O-])c1</smiles>

$\beta$-Chalcone derivative 1 (158)

Figure 14. Structures of some synthetic antibacterial chalcones.

\subsubsection{Antifungal Activity}

Chalcones are known to possess antifungal properties. One of the early studies on antifungal activities of naturally occurring chalcones was conducted by Jayasinghe et al. [73] (Table 2). In that work, several chalcones (79-81, 83 and 85) were isolated from the leaves of Artocarpus nobilis, and assessed for fungicidal activities against Cladosporium cladosporioides. All those chalcones showed fungicidal activity $(2-15 \mu \mathrm{g} / \mathrm{spot})$ in the thin layer chromatography (TLC) bio-authography method. 1-(5,7-Dihydroxy-2,2,6-trimethyl-2H-1-benzopyran8-yl)-3-phenyl-2-propen-1-one [58], 4'-hydroxyrottlerin (64), kamalachalcone E (67) and rottlerin (65), isolated from the fruits of Mallotus philippinensis, were tested for antifungal activity against several human pathogenic fungus [72]. Chalcone 58 and the chalcone dimer 67 showed significant antifungal properties $\left(\mathrm{IC}_{50}=4-16 \mu \mathrm{g} / \mathrm{mL}\right)$ against Aspergillus fumigates and Cryptococcus neoformans. Both compounds were particularly effective against Cryptococcus neoformans $\left(\mathrm{IC}_{50}=4 \mu \mathrm{g} / \mathrm{mL}\right)$. However, none of those chalcones displayed any noticeable antifungal activity against Aspergillus flavus, $A$. niger, Candida albicans, C. glabrata, and C. tropicalis [72]. 3,2'-Dihydroxy-2,4,4',6'-tetramethoxychalcone (55), 2'-hydroxy-2,4,4', $6^{\prime}$ tetramethoxychalcone (56), and $2^{\prime}$-hydroxy-2,3,4,4', $6^{\prime}$-pentamethoxychalcone (57) were shown active against $C$. albicans with MIC values in the range of $250-500 \mu \mathrm{g} / \mathrm{mL}$ [70].

These initial findings on antifungal properties of natural chalcones led the way to bioinspired synthesis of several other chalcone analogs with antifungal activities. Just over two decades ago, Lopez et al. [98] synthesized 41 chalcone derivatives, 11 of which (1, 159-168) (Figure 15) were found active against dermatophytes, e.g., Epidermophyton floccosum, Microsporum canis, M. gypseum, Trichophyton mentagrophytes and T. rubrum (MIC $=1.5-12.5 \mu \mathrm{g} / \mathrm{mL})$ These chalcones $(\mathbf{1}, \mathbf{1 5 9}-\mathbf{1 6 8})$ showed inhibitory properties against polymers of the fungal cell wall. However, none of these chalcones was active against Aspergillus niger, A. fumigatyus, A. flavus, Candida albicans, Cryptococcus neoformans and Saccharomyces cerevisiae. Antifungal activity of 12 further synthetic chalcones, albeit some of them are actually natural chalcones, were tested against Penicillium chrsogenum, A. niger, A. fluvus and Anthrobortys oligospora, and (E)-1-(4-hydroxyphenyl)-3-phenylprop-2-en-1-one (21), were found active against $P$. chrsogenum and $A$. nigar, (E)-1-(4-hydroxyphenyl)-3-(4methoxyphenyl)prop-2-en-1-one (169) displayed fungicidal effect against $P$. chrsogenum 
and 1-(4-hydroxyphenyl)-3-(4-methoxyphenyl)propan-1-one (170) was active against $A$. niger [99] (Figure 15).<smiles>[R]c1cc(/C=C/C(=O)c2ccccc2)c([R])c([R])c1[R]</smiles>

Chalcone (1) $\mathrm{R}=\mathrm{R}^{\prime}=\mathrm{R}^{\prime \prime}=\mathrm{R}^{\prime \prime \prime}=\mathrm{H}$

2,4-Dichloro-chalcone (159) $\mathrm{R}=\mathrm{R}^{\prime \prime}=\mathrm{Cl} ; \mathrm{R}^{\prime}=\mathrm{R}^{\prime \prime}=\mathrm{H}$

2,3-Dimethoxychalcone (160) $\mathrm{R}=\mathrm{R}^{\prime}=\mathrm{OMe} ; \mathrm{R}^{\prime \prime}=\mathrm{R}^{\prime \prime \prime}=\mathrm{H}$

2,4-Dimethoxychalcone (161) $\mathrm{R}^{\prime}=\mathrm{R}^{\prime \prime \prime}=\mathrm{H} ; \mathrm{R}=\mathrm{R}^{\prime \prime}=\mathrm{OMe}$

3,4-Dimethoxychalcone (162) $\mathrm{R}=\mathrm{R}^{\prime \prime \prime}=\mathrm{H} ; \mathrm{R}^{\prime}=\mathrm{R}^{\prime \prime}=\mathrm{OMe}$

3-Methoxychalcone (163) $\mathrm{R}=\mathrm{R}^{\prime \prime}=\mathrm{R}^{\prime \prime \prime}=\mathrm{H}$; $\mathrm{R}^{\prime}=\mathrm{OMe}$

4-Methoxychalcone (164) $\mathrm{R}=\mathrm{R}^{\prime}=\mathrm{R}^{\prime \prime \prime}=\mathrm{H} ; \mathrm{R}^{\prime \prime}=\mathrm{OMe}$

4-Methylchalcone (165) $\mathrm{R}=\mathrm{R}^{\prime}=\mathrm{R}^{\prime \prime \prime}=\mathrm{H} ; \mathrm{R}^{\prime \prime}=\mathrm{Me}$

2-Nitro-chalcone (166) $\mathrm{R}=\mathrm{NO}_{2} ; \mathrm{R}^{\prime}=\mathrm{R}^{\prime \prime}=\mathrm{R}^{\prime \prime \prime}=\mathrm{H}$

4-Nitro-chalcone (167) $\mathrm{R}=\mathrm{R}^{\prime}=\mathrm{R}^{\prime \prime \prime}=\mathrm{H} ; \mathrm{R}^{\prime \prime}=\mathrm{NO}_{2}$<smiles>COc1cccc(/C=C/C(=O)c2ccc(Br)cc2)c1</smiles>

4'-Bromo-4-methoxychalcone (168)<smiles>COc1ccc(CCC(=O)c2ccc(O)cc2)cc1</smiles>

1-(4-Hydroxyphenyl)-3-(4-methoxyphenyl)propan-1-one (170)<smiles>COc1ccc(/C=C/C(=O)c2ccc(O)cc2)cc1</smiles>

(E)-1-(4-Hydroxyphenyl)-3-(4-methoxyphenyl)prop-2-en-1-one (169)<smiles>COc1cc(C=C2CCc3ccccc3C2=O)cc(OC)c1OC</smiles>

(E)-2-(3',4',5'-Trimethoxy-benzylidene)-1tetralone (171)<smiles>[R]c1ccc(/C=C2\CCc3ccccc3C2=O)c([R])c1[R]</smiles>

(E)-2-(3',4'-Dimethoxy-benzylidene)-1-tetralone (31) $\mathrm{R}=\mathrm{H} ; \mathrm{R}^{\prime}=\mathrm{R}^{\prime \prime}=\mathrm{OMe}$

(E)-2-Benzylidene-1-tetralone (172) $\mathrm{R}=\mathrm{R}^{\prime}=\mathrm{R}^{\prime \prime}=\mathrm{H}$

(E)-2-(2'-Chloro-benzylidene)-1-tetralone (173) $\mathrm{R}=\mathrm{Cl} ; \mathrm{R}^{\prime}=\mathrm{R}^{\prime \prime}=\mathrm{H}$

(E)-2-(3'-Chloro-benzylidene)-1-tetralone (174) $\mathrm{R}=\mathrm{R}^{\prime \prime}=\mathrm{H} ; \mathrm{R}^{\prime}=\mathrm{Cl}$

(E)-2-(4'-Chloro-benzylidene)-1-tetralone (175) $\mathrm{R}=\mathrm{R}^{\prime}=\mathrm{H} ; \mathrm{R}^{\prime \prime}=\mathrm{Cl}$

(E)-2-(4'-Bromo-benzylidene)-1-tetralone (176) $\mathrm{R}=\mathrm{R}^{\prime}=\mathrm{H} ; \mathrm{R}^{\prime \prime}=\mathrm{Br}$

(E)-2-(4'-Fluoro-benzylidene)-1-tetralone (177) $\mathrm{R}=\mathrm{R}^{\prime}=\mathrm{H} ; \mathrm{R}^{\prime \prime}=\mathrm{F}$

(E)-2-(4'-Methoxy-benzylidene)-1-tetralone (178) $\mathrm{R}=\mathrm{R}^{\prime}=\mathrm{H} ; \mathrm{R}^{\prime \prime}=\mathrm{OMe}$

(E)-2-(2', $4^{\prime}$-Dichloro-benzylidene)-1-tetralone (179) $\mathrm{R}=\mathrm{R}^{\prime \prime}=\mathrm{Cl} ; \mathrm{R}^{\prime}=\mathrm{H}$

Figure 15. Structures of some synthetic antifungal chalcones.

Gupta et al. [100] synthesized 10 chalcone derivatives (31, 171-177) (Figure 15) and tested for their antifungal properties. None of these chalcones were active against $A$. niger and C. albicans, but showed strong fungicidal activity (MIC $=1.5-50.0 \mu \mathrm{g} / \mathrm{mL}$ ) against the dermatophyte Microsporum gypseum. It is noteworthy that (E)-2-benzylidene-1-tetralone (172) and (E)-2-(4'-chloro-benzylidene)-1-tetralone (175) exhibited better antifungal activity 
than the positive control ketoconazole, a well-known antifungal drug. It appeared that the addition of a $\mathrm{Cl}$ functionality at $\mathrm{C}-4^{\prime}$ of these compounds could enhance the antifungal potency. There are several other publications available to date that describe the synthesis and antifungal activity of chalcone derivatives having versatile chemical features [101-104].

\subsubsection{Antiviral Activity}

Chalcones were demonstrated to possess antiviral properties, which is thought to be exerted mainly by the disruption of the different stage of viral replication cycle, the inhibition of viral or cell enzymes, and/or the induction of apoptosis. One of the earliest studies on the antiviral activities of naturally occurring chalcones was conducted about two decades ago by Ochiumi et al. [76] (Table 2). In that study, naturally occurring chalcones, including licochalcone A (40), licochalcone B (68), and tetrahydroxy-methoxychalcone (69), were found to suppress TPA (12-O-tetradecanoylphorbol-13-acetate)-induced HIV promoter activity, and it was suggested that these chalcones could be used as templates for the development of novel anti-HIV drugs. Later, licochalcone A (40), licochalcone D (72), and licochalcone $G$ (73), isolated from G. inflata, were reported to possess antiviral properties against influenza virus [75]. Similarly, echinantin (59) and isoliquiritigenin (22) from the same plant were also active against this virus. The antiviral activity was measured by their noncompetitive neuraminidase inhibitory activity. Among these chalcones, echinantin (59) was the most potent one with an $\mathrm{IC}_{50}$ value in the range of $2.19-5.80 \mu \mathrm{g} / \mathrm{mL}$ against various strains of the influenza virus, e.g., H1N1, H9N2, novel H1N1 (WT), and oseltamivirresistant novel H1N1 (H274Y), expressed in 293T cells. In another study, dihydrochalcone diglycoside (50), isolated from the seagrass Thalassodendrin ciliatum, showed antiviral activity against the influenza A virus [74], and is assumed to have resulted from the electronic interactions between atomic charges within this compound in both aromatic rings and pseudo-receptor structures in the cells which prevent the attachment and penetration of the virus to the cell. Several alkylated chalcones, including xanthoangelol (83), xanthoangelols B, D-G (84-87), and xanthodeistal (88), isolated from Angelica keiskei (Table 2), were shown to possess significant antiviral properties [77], as evident from their inhibitory activity against cysteine proteases of SARS-CoV viruses. Among these compounds, xanthoangelol $\mathrm{E}$ (90), containing the perhydroxyl group, exhibited the most potent inhibitory activity against SARS-CoV 3CL ${ }^{\mathrm{PRO}}$ and $\mathrm{PL}^{\mathrm{PRO}}$ with $\mathrm{IC}_{50}$ values of 11.4 and $1.2 \mu \mathrm{M}$.

Promising antiviral properties of various naturally occurring chalcones tempted synthetic chemists to indulge in bioinspired synthesis of chalcone derivatives with potential antiviral properties. Such efforts have recently been further intensified with the emergence of various deadly respiratory viruses, and viral diseases including the current COVID-19 pandemic. Several studies have identified the activity of synthetic chalcones against different viruses. For example, Cole et al. [105] synthesized several O-benzyl-substituted chalcones, e.g., 1-(2-benzyloxy-6-hydroxyphenyl)-3-(5-bromo-2-methoxyphenyl)-propenone) (180), and determined their antiviral properties, suggesting that these compounds could be used for the prevention or treatment of human immunodeficiency virus (HIV) infections, whilst Mateeva et al. [106] investigated several synthetic chalcones (183-189) (Figure 16) as antiviral agents, and showed that all chalcones at a concentration of $5 \mu \mathrm{M}$ could inhibit, albeit at variable potencies, the translation of hepatitis $C$ virus. The inhibition of phosphorylated rsp6 (ribosomal protein S6) and S6K1 (ribosomal protein S6 kinase beta-1) by these chalcones could dampen hepatitis $C$ virus translation. This inhibition potentially was mediated through inhibition of mTOR (mechanistic target of rapamycin), which is a protein kinase that regulates cell growth, survival, and metabolism. 
<smiles>COc1ccc(Br)cc1/C=C/C(=O)c1c(O)cccc1OCc1ccccc1</smiles>

1-(2-Benzyloxy-6-hydroxyphenyl)-3-(5-bromo-2-methoxyphenyl)-propenone (180)<smiles>[R]c1ccc(OCC)c(/C=C/C(=O)c2c(O)cccc2OCc2ccccc2)c1</smiles>

1-(2-Benzyloxy-6-hydroxyphenyl)-3-(5-bromo-2-ethoxyphenyl)-propenone (181)

1-(2-Benzyloxy-6-hydroxyphenyl)-3-(5-chloro-2-ethoxyphenyl)-propenone (182)<smiles>[R]c1c(OC)cc(OC)c(C(=O)/C=C/c2cc(OC)c(OC)c(OC)c2)c1OC</smiles>

2',3,4,4',5-Pentamethoxy-6-hydroxychalcone (183) $\mathrm{R}=\mathrm{H}$

2',3,4,4',5-Pentamethoxy-5'-methyl-6-hydroxychalcone (184) $\mathrm{R}=\mathrm{Me}$

2',3,4,4',5-Pentamethoxy-5'-hexyl-6-hydroxychalcone (185) $\mathrm{R}=\mathrm{C}_{6} \mathrm{H}_{11}$<smiles>[R]c1cc([R])c(O)c(C(=O)/C=C/c2ccc(OC)c(OC)c2)c1</smiles>

3',5'-Dibromo-3,4-dimethoxy-6'-hydorxychalcone (186) $\mathrm{R}=\mathrm{R}^{\prime}=\mathrm{Br}$

3',5'-Dichloro-3,4-dimethoxy-6'-hydorxychalcone $(\mathbf{1 8 7}) \mathrm{R}=\mathrm{R}^{\prime}=\mathrm{Cl}$

3'-Methyl-5'-nitro-3,4-dimethoxy-6'-hydorxychalcone (188) $\mathrm{R}=\mathrm{NO}_{2} ; \mathrm{R}^{\prime}=\mathrm{Me}$<smiles>COc1cc(/C=C/C(=O)c2cc(Cl)cc(Cl)c2O)cc(OC)c1OC</smiles>

3',5'-Dichloro-3,4,5-trimethoxy-6'-hydorxychalcone (189)<smiles>CCOC(=O)C(C(=O)OCC)C(CC(=O)c1ccc(OCCSc2ncnc3[nH]cnc23)cc1)c1ccc(F)cc1</smiles>

Chalcone derivative containing a purine ether functionality (190)

Figure 16. Structures of some synthetic antiviral chalcones.

1-(2-Benzyloxy-6-hydroxyphenyl)-3-(5-bromo-2-methoxyphenyl)-propenone (180) was found to be the most potent as an antiviral agent, and could inhibit several clinical isolates of HIV in a dose-dependent fashion with $\mathrm{IC}_{50}$ values of around $5 \mu \mathrm{M}$ [105]. This finding led to further modification of this structure (180) to enhance antiviral potency and reduce toxicity towards normal human cells, which resulted in the synthesis of two more antiviral chalcones (181) and (182). Both chalcones (181 and 182) at a concentration of $10 \mu \mathrm{M}$ offered $>92 \%$ inhibition of viral propagation without impacting host cell viability. 
It appeared that halogenation at C-5 is an essential requirement for the activity of these benzoyloxychalcones. An ethoxy substituent in (181) and (182), instead of a methoxy in 180, offered increased efficacy, and a balance between high antiviral potency and low toxicity to host cells.

Most recently, Fu et al. [107] synthesized 28 chalcone derivatives containing a purine ether functionality, and derivative (190) (Figure 16) emerged as the best candidate to effectively inhibit the infectivity of tobacco mosaic virus in vivo with an $\mathrm{EC}_{50}$ value of $65.8 \mu \mathrm{g} / \mathrm{mL}$. It was demonstrated that this chalcone derivative could destroy the integrity of tobacco mosaic virus. A docking study suggested that the antiviral activity of (190) might depend on its strong binding affinity to tobacco mosaic virus coat protein (TMV-CP). There are several other reports on antiviral properties of synthetic chalcone derivatives available in the literature to date, including a few excellent review articles on this topic [108-110].

\subsection{Antioxidant Activity}

Antioxidants are compounds that can stop or prevent oxidative processes. They can be natural compounds as well as synthetic products. Antioxidants are effective in the management of oxidative stress, which is considered as one of the major causes of several chronic and life-threatening diseases. External supply of antioxidants is important for human health, and often achieved through regular diets containing plenty of fruits and vegetables. However, there are times when the additional supply of antioxidants in formulated forms may become essential. Plants have long been known as a source of antioxidants, and the phenolic and polyphenolic compounds are a major class of natural antioxidants. Most of the naturally occurring chalcones are phenolic compounds, which possess significant free-radical-scavenging and antioxidant properties. For example, lichochalcone C (71) [67] from Glycyrrhiza glabra and naringenin chalcone (78) and its glucoside, isosalipurposide (66), from Acacia cyanophylla [50] were shown to possess antioxidant properties (Table 2). Similarly, several chalcones (79-81, 83, and 85) were isolated from the leaves of Artocarpus nobilis, and were demonstrated to have antioxidant activity [73]. The antioxidant activity of isosalipurposide (66) was established by the 1,1-diphenyl-2-hydrazyl (DPPH) assay, the 2,2'-azino-bis(3-ethylbenzothiazoline-6-sulfonate) (ABTS) cation radical-scavenging assay, and the reducing power assay. The aglycone of glycoside 66, commonly known as naringenin chalcone (78), could have enhanced antioxidant potency because of the presence of an additional phenolic hydroxyl group. Additionally, this chalcone (66) was shown active against acetylcholinesterase with an $\mathrm{IC}_{50}$ value of $52.04 \mu \mathrm{g} / \mathrm{mL}$ [50]. Lichochalcone C (71) at a concentration of $50 \mu \mathrm{M}$ was found to significantly influence the antioxidant network activity of superoxide dismutase (SOD), catalase (CAT) and glutathione peroxidase (GPX) activity [67]. The geranylated chalcones (79-81, 83, and 85) showed strong qualitative radical-scavenging activity in the TLC-based DPPH assay [73].

Both the naturally occurring chalcones, and several synthetic chalcones, and their derivatives, were shown to possess antioxidant properties of varied potencies based on structural variations and diversity. Most recently, from a series of synthetic $\beta$-chalcone derivatives, chalcone (148) (Figure 14) was studied for its antioxidant activity using the DPPH and hydrogen-peroxide $\left(\mathrm{H}_{2} \mathrm{O}_{2}\right)$ assays [92]; this chalcone showed significant antioxidant (radical-scavenging) activity with the $\mathrm{IC}_{50}$ values of 298 and $530 \mu \mathrm{g} / \mathrm{mL}$, respectively, in the DPPH and $\mathrm{H}_{2} \mathrm{O}_{2}$ assays. Wang et al. [111] synthesized 41 chalcone derivatives and assessed their dual antioxidant mechanisms. Among the compounds, chalcone 191 (Figure 17) was found to be the most potent one, and showed cytoprotection of $\mathrm{H}_{2} \mathrm{O}_{2}$-induced oxidative damage in phaeochromocytoma (PC12) cells through freeradical-scavenging (as confirmed from the DPPH assay) and activation of the Nrf2/ARE antioxidant pathway at the same time. This chalcone (191) also displayed a noticeable effect against ischemia/reperfusion-related brain injury in animals. It was suggested that 2,5-dimethoxy-3' $4^{\prime}$-dihydroxychalcone (191) could act through dual antioxidant mechanisms, and could be considered as a direct and indirect antioxidant. A total of 26 chalcone derivatives with alkyl-substituted pyrazine heterocycle were synthesized and assessed for 
their radical-scavenging as well as cellular antioxidant capacity, impacting the growth of cells exposed to $\mathrm{H}_{2} \mathrm{O}_{2}$ [112]. Three of these synthetic chalcone analogs (192-194) (Figure 17) exhibited DPPH-radical-scavenging activity $\left(\mathrm{IC}_{50}=39-186 \mu \mathrm{M}\right)$ through a single electron transfer followed by a proton transfer mechanism, as revealed in the density functional theory (DFT) modeling. Clearly, chalcone 193 was the most active free-radical-scavenger with an $\mathrm{IC}_{50}$ value of $39 \mu \mathrm{M}$ in the DPPH assay. Earlier, Lahsasni et al. [113] synthesized a library of chalcone derivatives and chalcone 195 emerged as the most potent antioxidant among the analogs, and the activity was better than that of the well-known antioxidant ascorbic acid in the DPPH assay.<smiles>COc1ccc(OC)c(/C=C/C(=O)c2ccc(O)c(O)c2)c1</smiles>

2,5-Dimethoxy-3'4'-dihydroxychalcone (191)<smiles>[R]c1cnc(C(=O)/C=C/c2ccc(O)c(OC)c2)cn1</smiles>

Chalcone-pyrazine analog a (192) $\mathrm{R}=\mathrm{H}$

Chalcone-pyrazine analog b (193) $\mathrm{R}=$ Isopropyl

Chalcone-pyrazine analog c (194) R = Isobutyl<smiles>CCCCCCCCCCCCCCCC(=O)Oc1cccc(/C=C/C(=O)c2ccc([N+](=O)[O-])cc2)c1</smiles>

Chalcone long-chain fatty acid ester (195)

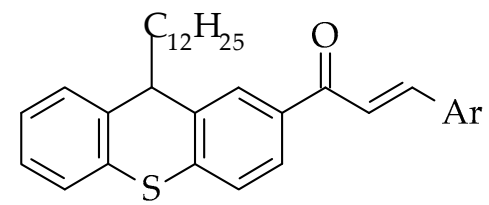

Chacone-phenothoazine derivative a (196) Ar = 4-Dimethyl-aminophenyl

Chacone-phenothoazine derivative b (197) Ar = 4-Chlorophenyl

Chacone-phenothoazine derivative c (198) Ar = 4-Methoxyphenyl

Chacone-phenothoazine derivative d (199) Ar = 3,4-Dimethoxyphenyl

Chacone-phenothoazine derivative e (200) Ar = 2-Furfuyl

Chacone-phenothoazine derivative $\mathrm{f}$ (201) $\mathrm{Ar}=3$-Thienyl

Chacone-phenothoazine derivative $\mathrm{g}$ (202) $\mathrm{Ar}=3,4,5$-Trimethoxyphenyl

Figure 17. Structures of some synthetic antioxidant chalcones.

2'-Hydroxy-4-methoxychalcone (139), a recently synthesized chalcone, showed a considerable antioxidant activity [89]. It was found to be able to attenuate LPS-induced oxidative stress and promote antioxidant defense in RAW264.7 macrophages. 2'-Hydroxy4-methoxychalcone pretreatment at a concentration range of $0.01-1.0 \mu \mathrm{M}$ could augment the nuclear expression of Nrf2 in a concentration-dependent manner. An increased level of the downstream antioxidant protein heme oxygen SE-1 was also observed. Additionally, this pretreatment was found to significantly increase the levels of non-enzymatic antioxidant GSH (glutathione) in LPS-stimulated RAW 264.7 cells. Zahrani et al. [114] reported the synthesis of several chalcone-based phenothiazine derivatives and their antioxidant potentials based on the DPPH assay; seven of these compounds (196-202) showed promising DPPH-radical-scavenging activity. There are several other reports depicting the synthesis and antioxidant properties of chalcone derivatives available in the literature, most of which possess DPPH-radical-scavenging properties [115-118]. 


\subsection{Antiparasitic Activity}

Parasites are organisms that live and feed on another living being, e.g., animals, humans, insects, or plants, and most often cause harm to the host organism. Parasitic diseases, such as like leishmaniasis and malaria, remain a major global health concern, and research consequently continues in the search for effective and affordable drugs to combat various parasitic diseases. Some naturally occurring chalcones were shown to possess antiparasitic activities (Table 2). For example, flavokawain B (28), isolated from Polygonum ferrugineum [79]; (E)-1-(2,4-dihydroxy-3-(3-methylbut-2-en-1-yl)phenyl)-3-phenylprop-2-en-1one (53) from Lonchocarpus sp. [78]; licoagrochalcone A (70) from Erythrina abyssinica [81]; and licochalcone C (71) isolated from Glycyrrhiza glabra [80] were found to possess significant antiparasitic/antiprotozoal properties. A protozoa can be free-living or parasitic. Most of the disease-causing protozoa are parasitic. Even free-living protozoa when entering other cells and tissues of living being become parasitic. While licochalcone C (71) was found active against malarial parasite Plasmodium falciparum, chalcone $\mathbf{5 3}$ showed activity against Leishmania and Trypanosoma species. Flavokawain B (28) demonstrated trypanocidal activity against Trypanosoma cruzi and T. bruceii, with $\mathrm{IC}_{50}$ values of $9.5 \mu \mathrm{M}$ and $4.8 \mu \mathrm{M}$, respectively [79], whereas licoagrochalcone A (70) was shown to have antiplasmodial activity against chloroquine-sensitive and chloroquin-resistant strains of Plasmodium falciparum; the $\mathrm{IC}_{50}$ values were determined as 12.7 and $12.0 \mu \mathrm{M}$, respectively [81]. Two other chalcones from Erythrina abyssinica, homobutein (19) $\left(\mathrm{IC}_{50}=15.0\right.$ and $16.1 \mu \mathrm{M}$, respectively) and 5-prenylated derivative of butein (16) ( $\mathrm{IC}_{50}=10.3$ and $11.2 \mu \mathrm{M}$, respectively) were also active against both strains for Plasmodium falciparum.

Inspired by the observed antiparasitic activity of naturally occurring chalcones, several synthetic chalcones with potential antiparasitic activity were introduced. Ugwu et al. [119] reported that several synthetic chalcone derivatives of the structural classes chromanochalcones, chromeno-dehydrochalcones, quinolinyl chalcones, morachalcones, prenylated chalcones, chromenochalcones, quinoxaline chalcones, chalcone sulphonamides, and licochalcones could exhibit antimalarial activity, and some of them were even active against chloroquin-resistant $P$. falciparaum. Structures of some of those antimalarial chalcone derivatives (203-207) are presented in Figure 18. Yadav et al. [120] synthesized 27 antimalarial chalcones, and from the antiplasmodial screening of these compounds, chalcone derivative 1-(4-benzimidazol-1-yl-phenyl)-3-(2, 4-dimethoxy-phenyl)-propen-1-one (208) appeared as the most potent one with an $\mathrm{IC}_{50}$ value of $1.1 \mu \mathrm{g} / \mathrm{mL}$ against $P$. falciparum. Among the 27 compounds tested, the presence of two methoxyl functionalities at positions 2 and 4 appeared to be optimum for antimalarial activity followed by 3,4-dimethoxy and 2,5dimethoxy with moderate activity. It was noted that 3,4,5-trimethoxy series of derivatives displayed weak activity, probably because of the stearic hindrance at the binding site of the enzyme. Several dimeric chalcone derivatives were synthesized and their antimalarial potential was evaluated using in vitro globin hydrolysis, $\beta$-hematin formation, and murine Plasmodium berghei [121]. Among the deimeric chalcone derivatives, 1,1-bis-[(3', 4'$\mathrm{N}$-(urenylphenyl)-3-(3", $4^{\prime \prime}, 5^{\prime \prime}$-trimethoxyphenyl)]-2-propen-1-one (209) (Figure 18) was found to be the most active antimalarial candidate.

Quinolinone-chalcone derivatives were synthesized and assessed for their antiparasitic activity against the mammalian stages of Trypanosoma brucei and Leishmania infantum [122]. Among the compounds, quinolinone-chalcone derivative $\mathbf{2 1 0}$ exhibited the most prominent activity against both parasites, particularly against Leishmania infantum $\left(\mathrm{IC}_{50}=1.3 \mu \mathrm{M}\right)$. Another derivative (211) (Figure 18) showed significant trypanocidal activity $\left(\mathrm{IC}_{50}=2.6 \mu \mathrm{M}\right)$. These are just a few examples of antiparasitic synthetic chalcone derivatives, and there are many more examples are available in the literature [123,124]. 
<smiles>O=C(/C=C(\I)c1cc2ccccc2nc1Cl)c1cc(Br)cc(Cl)c1O</smiles>

Antimalarial quinolinyl chalcone (203)<smiles>CC(C)=CCCC1(C)CCc2c(O)c(C(=O)/C=C/c3cc(C)ccc3C)cc(CC=C(C)C)c2O1</smiles>

Antimalarial chromenochalcone (205)<smiles>COc1cc(C2CC2C(=O)c2nc3cc(O)c(O)cc3nc2C)cc(OC)c1OC</smiles>

Antimalarial quinoxaline chalcone (207)<smiles>CC(C)=CCCC1(C)CCc2c(ccc(C(=O)/C=C/c3ccc(O)cc3)c2O)O1</smiles>

Antimalarial prenylated chalcone (204)<smiles>CC(C)=CCCC1(C)CCc2ccc(O)c(C(=O)CCc3cc(C=O)ccc3O)c2O1</smiles>

Antimalarial chromeno-dihydrochalcone (206)<smiles>COc1ccc(/C=C/C(=O)c2ccc(-c3nc4ccccc4n3C)cc2)c(OC)c1</smiles>

1-(4-Benzimidazol-1-yl-phenyl)-3-(2, 4-dimethoxyphenyl)-propen-1-one (208)<smiles>COc1ccc(/C=C/C(=O)c2ccc(NC(=O)Nc3cccc(C(=O)/C=C/c4ccc(OC)cc4OC)c3)cc2)c(OC)c1</smiles>

1,1-Bis-[(3',4'-N-(urenylphenyl)-3-(3",4",5"-trimethoxyphenyl)]-2-propen-1-one (209)<smiles>CC(C)(C)c1cc(/C=C/C(=O)c2c(O)c3ccccc3[nH]c2=O)cc(C(C)(C)C)c1O</smiles>

Quinolinone-chalcone derivative (210)<smiles>Cc1ccccc1/C=C/C(=O)c1c(O)c2ccccc2[nH]c1=O</smiles>

Trypanocidal quinolinone-chalcone derivative (210)

Figure 18. Structures of some synthetic antiparasitic chalcones.

\subsection{Immunoregulatory Activity}

Natural chalcones mallotophilippens (74-76) displayed immunoregulatory activity [68]. The immunomodulatory activity of synthetic sulfonamide chalcone derivatives in mice infected with filarial parasites, including Brugia malayi, has recently been studied [125]. Lee et al. [126] reviewed the potential immunomodulatory effects of natural and synthetic chalcones, and several synthetic chalcone derivatives were reported to possess immunomodulatory properties, mediated through multiple mechanisms, e.g., actions on 
dendritic cells, neutrophils, basophils, innate lymphoid cells, microglial cells, and T cells. Figure 19 shows some examples of synthetic immunomodulatory chalcone derivatives.<smiles>O=C(/C=C/c1ccc2ccccc2c1)c1cc(O)ccc1O</smiles>

2',5'-Dihydroxy2-naphthylchalcone (211)<smiles>COc1cc(O)c(C(=O)/C=C/c2ccccn2)cc1CN1CCOCC1</smiles>

E)-1-[4-ethoxy-2-hydroxy-5-(morpholinomethyl)phenyl]-3-(pyridin-2-yl)prop2-en-1-one (213)<smiles>O=C(/C=C/c1ccco1)c1cc(O)ccc1O</smiles>

2',5'-Dihydroxy-2-furfurylchalcone (215)<smiles>COc1ccc(C(=O)/C=C/c2ccccn2)c(O)c1CN1CCOCC1</smiles>

(E)-1-[2-Hydroxy-4-methoxy-3-(morpholinomethyl)phenyl]-3-(pyridin-2-yl)prop-2-en-1-one (212)<smiles>COc1ccc(C(=O)/C=C/c2cc3ccccc3nc2Cl)c(OC)c1OC</smiles>

1-(2,3,4-Trimethoxyphenyl)-3-[3-(2-chloroquinolinyl)]-2-propen-1-one (214)<smiles>COCOCCc1cc(OCOC)cc(OCOC)c1CCC(=O)C#Cc1ccccc1</smiles>

3-Phenyl-1-(2,4,6-tris(methoxymethoxy)-phenyl)prop-2-yn-1-one (216)<smiles>CC(C)CCOc1cccc(O)c1C(=O)/C=C/c1ccc(O)cc1</smiles>

2',4-Dihydroxy-6'-isopentyloxychalcone (217)

Figure 19. Structures of some synthetic immunomodulatory chalcones.

Synthetic chalcone analogs, including 2',5'-dihydroxy2-naphthylchalcone (211), could act on neutrophil degranulation and superoxide anion generation, while heterocyclic chalcones, namely (E)-1-[2-hydroxy-4-methoxy-3-(morpholinomethyl)phenyl]-3-(pyridin-2-yl)prop-2en-1-one (212) and (E)-1-[4-ethoxy-2-hydroxy-5-(morpholinomethyl)phenyl]-3-(pyridin-2yl)prop-2-en-1-one (213) (Figure 19), were found to inhibit the generation of superoxide anion and elastases [126]. Similarly, 1-(2,3,4-trimethoxyphenyl)-3-(3-(2-chloroquinolinyl)-2propen-1-one (214) could suppress the production of elastase and superoxide anion, and LTB4(leukotriene B4) release in human neutrophils. The $\mathrm{N}$-formylmethionyl-leucyl-phenylalanine (fMLP)-stimulated respiratory burst in neutrophils was found to be inhibited by the synthetic chalcone analog, 2', $5^{\prime}$-dihydroxy-2-furfurylchalcone (215). An unusual synthetic chalcone 
derivative, 3-phenyl-1-(2,4,6- tris(methoxymethoxy)phenyl)prop-2-yn-1-one (216), was shown to possess immunomodulatory properties, whereas 2',4-dihydroxy-6'-isopentyloxychalcone (217) could modulate an innate immune response [126]. These are just a few examples of synthetic chalcone derivatives that can modulate an immune response, and there are many more similar examples available in the literature [127].

\section{Conclusions}

Over the years, thousands of chalcones and their derivatives were isolated from natural sources, and many of those were screened for potential bioactivities, mainly including anticancer, anti-inflammatory, antimicrobial, antioxidant, and antiparasitic properties. The $\mathrm{d}$ of bioactive naturally occurring chalcones has inspired total or partial synthesis of chalcone analogs as well as minor structural modifications of natural chalcones forming a large collection of bioactive synthetic chalcone derivatives, some of which have enhanced bioactivities and/or reduced toxicities compared to relevant natural chalcones. This review article has demonstrated how bioinspired synthesis of chalcone derivatives can potentially introduce a new chemical space for the exploitation for new drug discovery.

Author Contributions: All authors contributed equally to collation of relevant information from extensive literature search. Additionally, L.N. and K.J.R. prepared, edited and submitted the manuscript as corresponding authors. All authors have read and agreed to the published version of the manuscript.

Funding: Lutfun Nahar (L.N.) gratefully acknowledges the financial support of the European Regional Development Fund-Project ENOCH (No. CZ.02.1.01/0.0/0.0/16_019/0000868).

Data Availability Statement: All relevant data were presented as an integral part of this manuscript.

Acknowledgments: Lutfun Nahar gratefully acknowledges the financial support of the European Regional Development Fund-Project ENOCH (No. CZ.02.1.01/0.0/0.0/16_019/0000868). The Government of Iraq is thanked for a PhD scholarship to Hiba Jasim.

Conflicts of Interest: The authors declare no conflict of interest.

\section{References}

1. Nahar, L.; Sarker, S.D. Chemistry for Pharmacy Students: General, Organic and Natural Product Chemistry, 2nd ed.; Wiley and Sons: Chichester, UK, 2019.

2. Zhuang, C.; Zhang, W.; Sheng, C.; Zhang, W.; Xing, C. Chalcone: A privileged structure in medicinal chemistry. Chem. Rev. 2017, 117, 7762-7810. [CrossRef]

3. Valavanidis, A.; Vlachogianni, T. Plant polyphenols: Recent advances in epidemiological research and other studies on cancer prevention. In Studies in Natural Products Chemistry; Atta-Ur-Rahman, T.I., Ed.; Elsevier: Amsterdam, The Netherlands, 2013; Volume 39, pp. 269-295.

4. Gupta, D.; Jain, D.; Trivedi, P. Recent advances in chalcones as anti-infective agents. Int. J. Chem. Sci. 2010, 8, 649-654.

5. Mahapatra, D.K.; Bharti, S.K.; Asati, V. Chalcone scaffolds as anti-infective agents: Structural and molecular target perspectives. J. Med. Chem. Eur. J. Med. Chem. 2015, 101, 496-524. [CrossRef]

6. Hutchins, W.A.; Wheeler, T.S. Chalkones: A new synthesis of chrysin, apigenin and luteolin. J. Chem. Soc. 1939, 91-94. [CrossRef]

7. Bohm, B.A. Chalcones and aurones-7. Methods Plant. Biochem. 1989, 1, 237-282.

8. Cazarolli, L.H.; Kappel, V.D.; Zanatta, A.P.; Suzuki, D.O.H.; Yunes, R.A.; Nunes, R.J.; Pizzolatti, M.G.; Silva, F.R.M.B. Natural and synthetic chalcones: Tools for the study of targets of action-Insulin secretagogue or insulin mimetic? In Studies in Natural Products Chemistry; Atta-Ur-Rahman, T.I., Ed.; Elsevier: Amsterdam, The Netherlands, 2013; Volume 39, pp. 47-89.

9. Watson, R.R. Nutrition and Functional Foods for Healthy Aging, 1st ed.; Elsevier Science: Amsterdam, The Netherlands, 2017; pp. 1-386.

10. Higgins, L.G. Induction of cancer chemopreventive enzymes by coffee is mediated by transcription factor Nrf2. Evidence that the coffee-specific diterpenes cafestol and kahweol confer protection against acrolein. Pharmacol. Toxicol. Appl. Pharmacol. 2008, 226, 328-337. [CrossRef] [PubMed]

11. Bryan, H.K.; Olayanju, A.; Goldring, C.E.; Park, B.K. The Nrf2 cell defence pathway: Keap1-dependent and -independent mechanisms of regulation. Biochem. Pharmacol. 2013, 85, 705-717. [CrossRef]

12. Thurston, D.E. Chemistry and Pharmacology of Anticancer Drugs; CRC Press Inc.: Boca Raton, FL, USA, 2006.

13. Zhang, Y.; Hou, Y.; Liu, C.; Li, Y.; Guo, W.; Wu, J.-L.; Xu, D.; You, X.; Pan, Y.; Chen, Y. Identification of an adaptor protein that facilitates Nrf2-Keap1 complex formation and modulates antioxidant response. Free Radic. Biol. Med. 2016, 97, 38-49. [CrossRef] 
14. Kim, H.J.; Jang, B.K.; Park, J.-H.; Choi, J.W.; Park, S.J.; Byeon, S.R.; Pae, A.N.; Lee, Y.S.; Cheong, E.; Park, K.D. A novel chalcone derivatives as Nrf2 activator attenuates learning and memory impairment in a scopolamine-induced mouse model. J. Med. Chem. Eur. J. Med. Chem. 2020, 185, 111777. [CrossRef]

15. Egbujor, M.C.; Saha, S.; Buttari, B.; Profumo, E.; Saso, L. Activation of Nrf2 signaling pathway by natural and synthetic chalcones: A therapeutic road map for oxidative stress. Expert Opin. Clin. Pharmacol. 2021, 14, 465-480. [CrossRef]

16. Ajiboye, T.O.; Yakubu, M.T.; Oladiji, A.T. Electrophilic and reactive oxygen species detoxification potentials of chalcone dimers is mediated by redox transcription factor Nrf-2. J. Biochem. Mol. Toxicol. 2014, 28, 11-22. [CrossRef] [PubMed]

17. Li, N.; Meng, D.; Pan, Y.; Cui, Q.; Li, G.; Ni, H.; Sun, Y.; Qing, D.; Jia, X.; Pan, Y. Anti-neuroinflammatory and NQO1 inducing activity of natural phytochemicals from Coreopsis tinctoria. J. Funct. Foods 2015, 17, 837-846. [CrossRef]

18. Martinez, R.M.; Pinho-Ribeiro, F.A.; Vale, D.L.; Steffen, V.S.; Vicentini, F.T.M.C.; Vignoli, J.A.; Baracat, M.M.; Georgetti, S.R.; Verri, W.A.; Casagrande, R. Trans.-chalcone added in topical formulation inhibits skin inflammation and oxidative stress in a model of ultraviolet B radiation skin damage in hairless mice. J. Photochem. Photobiol. 2017, 171, 139-146. [CrossRef]

19. Kachadourian, R.; Day, B.J.; Pugazhenti, S.; Franklin, C.C.; Genoux-Bastide, E.; Mahaffey, G.; Gauthier, C.; Di Pietro, A.; Boumendjel, A.N. A synthetic chalcone as a potent inducer of glutathione biosynthesis. J. Med. Chem. 2012, 55, 1382-1388. [CrossRef] [PubMed]

20. Basar, N.; Nahar, L.; Oridupa, O.A.; Ritchie, K.J.; Talukdar, A.D.; Stafford, A.; Kushiev, H.; Kan, A.; Sarker, S.D. Utilization of the ability to induce activation of the nuclear factor (erythroid-derived 2)-like factor 2 (Nrf2) to assess potential cancer chemopreventive activity of liquorice samples. Phytochem. Anal. 2016, 27, 233-238. [CrossRef]

21. Plopper, G.; Sharp, D.; Sikorski, E.; Lewin, B. Lewin's Cells, 3rd ed.; Plopper, G., Sharp, D., Sikorski, E., Eds.; Jones \& Bartlett Learning: Burlington, MA, USA, 2015.

22. Orlikova, B.; Schnekenburger, M.; Zloh, M.; Golais, F.; Diederich, M.; Tasdemir, D. Natural chalcones as dual inhibitors of HDACs and NF-кB. Oncol. Rep. 2012, 28, 797-805. [CrossRef]

23. Lee, Y.H.; Jeon, S.-H.; Kim, S.H.; Kim, C.; Lee, S.-J.; Koh, D.; Lim, Y.; Ha, K.; Shin, S.Y. A new synthetic chalcone derivative, 2-hydroxy-3', 5, 5'-trimethoxychalcone (DK-139), suppresses the Toll-like receptor 4-mediated inflammatory response through inhibition of the Akt/NF-kB pathway in BV2 microglial cells. Exp. Mol. Med. 2012, 44, 369-377. [CrossRef]

24. Zhong, P.; Wu, L.; Qian, Y.; Fang, Q.; Liang, D.; Wang, J.; Zeng, C.; Wang, Y.; Liang, G. Blockage of ROS and NF-kB-mediated inflammation by a new chalcone L6H9 protects cardiomyocytes from hyperglycemia-induced injuries. BBA Mol. Basis Dis. 2015, 1852, 1230-1241. [CrossRef]

25. Rajajendram, R.; Tham, C.L.; Akhtar, M.N.; Sulaiman, M.R.; Israf, D.A. Inhibition of epithelial CC-family chemokine synthesis by the synthetic chalcone DMPF-1 via disruption of NF-[kappa]B nuclear translocation and suppression of experimental asthma in mice. Mediat. Inflamm. 2015, 2015, 176926. [CrossRef]

26. Kuruc, T.; Kello, M.; Petrova, K.; Kudlickova, Z.; Kubatka, P. The newly synthesized chalcone L1 is involved in the cell growth inhibition, induction of apoptosis and suppression of epithelial-to-mesenchymal transition of HeLa cells. Molecules 2021, 26,1356 [CrossRef]

27. Bortolotto, L.F.B.; Barbosa, F.R.; Silva, G.; Bitencourt, T.A.; Beleboni, R.O.; Baek, S.J.; Marins, M.; Fachin, A.L. Cytotoxicity of trans-chalcone and lichochalcone A against breast cancer cells is due to apoptosis induction and cell cycle arrest. Biomed. Pharmacother. 2017, 85, 425-433. [CrossRef] [PubMed]

28. Qi, Z.; Liu, M.; Liu, Y.; Zhang, M.; Yang, G. Tetramethoxychalcone, a chalcone derivative, suppresses proliferation, block cell cycle progression, and induces apoptosis of human ovarian cancer cells. PLoS ONE 2014, 9, e106206. [CrossRef]

29. Hseu, Y.-C.; Lee, M.-S.; Wu, C.-R.; Cho, H.-J.; Lin, K.-Y.; Lai, G.-H.; Wang, S.-Y.; Kuo, Y.-H.; Kumar, K.J.S.; Yang, H.-L. The chalcone flavokawain B induces G2/M cell-cycle arrest and apoptosis in human oral carcinoma HSC-3 cells through the intracellular ROS generation and downregulation of the Akt/p38 MAOK signaling pathway. J. Agric. Food Chem. 2012, 60, 2385-2397. [CrossRef]

30. Ko, H.; Kim, Y.; Park, J.; Amor, E.C.; Lee, J.W.; Yang, H. Dimethyl cardamonin induces G(1)-phase cell cycle arrest, apoptosis, and autophagy in HCT116 cells. Cancer Res. 2010, 70, 780.

31. Maioral, M.F.; Gaspar, P.C.; Souza, G.R.R.; Mascarello, A.; Chiaradia, L.D.; Licínio, M.A.; Moraes, A.C.R.; Yunes, R.A.; Nunes, R.J.; Santos-Silva, M.C. Apoptotic events induced by synthetic naphthylchalcones in human acute leukemia cell lines. Biochimie 2013, 95, 866-874. [CrossRef] [PubMed]

32. Kello, M.; Drutovic, D.; Pilatova, M.B.; Tischlerova, V.; Perjesi, P.; Mojzis, J. Chalcone derivatives cause accumulation of colon cancer cells in the G2/M phase and induce apoptosis. Life Sci. 2016, 150, 32-38. [CrossRef] [PubMed]

33. Novilla, A.; Astuti, I.; Suwito, H. Molecular Mechanism of synthesized potential anticancer agent chalcone in leukemia cell line K562. J. Med. Sci. 2017, 49, 23-28.

34. Rao, Y.K.; Kao, T.-Y.; Ko, J.-L.; Tzeng, Y.-M. Chalcone HTMC causes in vitro selective cytotoxicity, cell-cycle G 1 phase arrest through p53-dependent pathway in human lung adenocarcinoma A549 cells, and in vivo tumor growth suppression. Bioorg. Med. Chem. Lett. 2010, 20, 6508-6512. [CrossRef] [PubMed]

35. Mohamed, M.F.; Hassaneen, H.M.; Abdelhamid, I.A. Cytotoxicity, molecular modeling, cell cycle arrest, and apoptotic induction induced by novel tetrahydro-[1,2,4]triazolo[3,4-a]isoquinoline chalcones. J. Med. Chem. Eur. J. Med. Chem. 2018, 143, 532-541. [CrossRef] 
36. Zhang, B.; Lai, Y.; Li, Y.; Shu, N.; Wang, Z.; Wang, Y.; Li, Y.; Chen, Z. Antineoplastic activity of isoliquiritigenin, a chalcone compound, in androgen-independent human prostate cancer cells linked to G2/M cell cycle arrest and cell apoptosis. Eur. J. Pharmacol. 2018, 821, 57-67. [CrossRef]

37. Takac, P.; Kello, M.; Pilatova, M.B.; Kudlickova, Z.; Vilkova, M.; Slepcikova, P.; Petik, P.; Mojzis, J. New chalcone derivative exhibits antiproliferative potential by inducing G2/M cell cycle arrest, mitochondrial-mediated apoptosis and modulation of MAPK signalling pathway. Chem. Interact. 2018, 292, 37-49. [CrossRef] [PubMed]

38. Wani, Z.A.; Pathania, A.S.; Mahajan, G.; Behl, A.; Mintoo, M.J.; Guru, S.K.; Viswanath, A.; Malik, F.; Kamal, A.; Mondhe, D.M. Anticancer activity of a novel quinazolinone-chalcone derivative through cell cycle arrest in pancreatic cancer cell line. J. Solid Tumors 2015, 5, 73-76. [CrossRef]

39. Elmore, S. Apoptosis: A review of programmed cell death. Toxicol. Pathol. 2007, 35, 495-516. [CrossRef] [PubMed]

40. Hsu, Y.; Kuo, P.; Tzeng, W.; Lin, C. Chalcone inhibits the proliferation of human breast cancer cell by blocking cell cycle progression and inducing apoptosis. Food Chem. Toxicol. 2006, 44, 704-713. [CrossRef]

41. Chen, G.; Zhou, D.; Li, X.-Z.; Jiang, Z.; Tan, C.; Wei, X.-Y.; Ling, J.; Jing, J.; Liu, F.; Li, N. A natural chalcone induces apoptosis in lung cancer cells: 3D-QSAR, docking and an in vivo/vitro assay. Sci. Rep. 2017, 7, 10729. [CrossRef]

42. Pedrini, F.S.; Licínio, M.A.; De Moraes, A.C.R.; Curta, J.C.; Costa, A.; Santos-Silva, M.C.; Chiaradia, L.D.; Mascarello, A.; Nunes, R.J.; Yunes, R.A.; et al. Induction of apoptosis and cell cycle arrest in L-1210 murine lymphoblastic leukaemia cells by (2E)-3-(2-naphthyl)-1-(3'-methoxy-4'-hydroxy- phenyl)-2-propen-1-one. J. Pharm. Pharmacol. 2010, 62, 1128-1136. [CrossRef]

43. Zhang, Y.; Srinivasan, B.; Xing, C.; Lü, J. A new chalcone derivative (E)-3-(4-methoxyphenyl)-2-methyl-1-(3,4,5-trimethoxyphenyl) prop-2-en-1-one suppresses prostate cancer involving p53-mediated cell cycle arrests and apoptosis. Anticancer Res. 2012, 32, 3689-3698.

44. Maggiolini, M.; Statti, G.; Vivacqua, A.; Gabriele, S.; Rago, V.; Loizzo, M.; Menichini, F.; Amdo, S. Estrogenic and antiproliferative activities of isoliquiritigenin in MCF-7 breast cancer cells. J. Steroid Biochem. Mol. Biol. 2002, 82, 315-322. [CrossRef]

45. Mutchtaridi, M.; Syahidah, H.N.; Subarans, A.; Yusuf, M.; Bryant, S.D.; Langer, T. Molecular docking and 3D-pharmacophore modeling to study the interactions of chalcone derivatives with estrogen receptor alpha. Pharmaceutical 2017, $10,81$.

46. Prasetiawati, R.; Zamri, A.; Barliana, M.I.; Mutchtaridi, M. In silico predictive for modification of chalcone with pyrazole derivatives as a novel therapeutic compound for targeted breast cancer treatment. J. Appl. Pharm. Sci. 2019, 9, 20-28.

47. Herber, C.B.; Quirti, J.G.; Firestone, G.; Krois, C. $2^{\prime}, 3^{\prime}, 4^{\prime}$-Trihydroxychalcone is an estrogen receptor ligand which modulates the activity of 17ß-estradiol. bioRxiv 2019, 607275. [CrossRef]

48. Branham, W.S.; Dial, S.L.; Moland, C.L.; Hass, B.S.; Blair, R.M.; Fang, H.; Shi, L.; Tong, W.; Perkins, R.G.; Sheehan, D.M. Phytoestrogens and mycoestrogens bind to the rat uterine estrogen receptor. J. Nutr. 2002, 132, 658-664. [CrossRef] [PubMed]

49. Dube, P.N.; Thombare, Y.B.; Chatpalliwar, V.A. Design and Synthesis of Novel chalcone-phenylpyranone derivatives as estrogen receptor modulators. Proceedings 2018, 9, 30. [CrossRef]

50. Ghribia, L.; Ghouilaa, H.; Omrib, A.; Besbesb, M.; Janneta, H.B. Antioxidant and anti-acetylcholinesterase activities of extracts and secondary metabolites from Acacia cyanophylla. Asian Pac. J. Trop. Biomed. 2014, 4, S417-S423. [CrossRef] [PubMed]

51. Voon, F.-L.; Sulaiman, M.R.; Akhtar, M.N.; Idris, M.F.; Akira, A.; Perimal, E.K.; Israf, D.A.; Ming-Tatt, L. Cardamonin (2', 4' dihydroxy-6'-methoxychalcone) isolated from Boesenbergia rotunda (L.) Mansf. inhibits CFA-induced rheumatoid arthritis in rats. Eur. J. Pharmacol. 2017, 794, 127-134. [CrossRef] [PubMed]

52. Choi, H.S.; Kim, M.K.; Choi, Y.K.; Shin, Y.C.; Cho, S.-G.; Ko, S.-G. Rhus verniciflua Stokes (RVS) and butein induce apoptosis of paclitaxel-resistant SKOV-3/PAX ovarian cancer cells through inhibition of AKT phosphorylation. BMC Complement. Altern. Med. 2016, 16, 122. [CrossRef] [PubMed]

53. Rozmer, Z.; Perjesi, P. Naturally occurring chalcones and their biological activities. Phytochem. Rev. 2016, 15, 87-120. [CrossRef]

54. Akihisa, T.; Tokuda, H.; Hasegawa, D.; Ukiya, M.; Kimura, Y.; Enjo, F.; Suzuki, T.; Nishino, H. Chalcones and other compounds from the exudates of Angelica keiskei and their cancer chemopreventive effects. Indian J. Nat. Prod. 2006, 69, 38-42. [CrossRef]

55. Memon, A.H.; Ismail, Z.; Aisha, A.F.; Al-Suede, F.S.R.; Hamil, M.S.R.; Hashim, S.; Saeed, M.A.A.; Laghari, M.; Majid, A.; Shah, A.M. Isolation, characterization, crystal structure elucidation, and anticancer study of dimethyl cardamonin, isolated from Syzygium campanulatum Korth. Evid. Based Complement. Alternat. Med. 2014, 2014, 470179. [CrossRef]

56. Abu, N.; Akhtar, M.N.; Yeap, S.K.; Lim, K.L.; Ho, W.Y.; Abdullah, M.P.; Ho, C.L.; Omar, A.R.; Ismail, J.; Alitheen, N.B. Flavokawain B induced cytotoxicity in two breast cancer cell lines, MCF-7 and MDA-MB231 and inhibited the metastatic potential of MDAMB231 via the regulation of several tyrosine kinases In vitro. BMC Complement. Altern. Med. 2016, 16, 86. [CrossRef]

57. Kuo, Y.-F.; Su, Y.-Z.; Tseng, Y.-H.; Wang, S.-Y.; Wang, H.-M.; Chueh, P.J. Flavokawain B, a novel chalcone from Alpinia pricei Hayata with potent apoptotic activity: Involvement of ROS and GADD153 upstream of mitochondria-dependent apoptosis in HCT116 cells. Free Radic. Biol. Med. 2010, 49, 214-226. [CrossRef]

58. Yang, L.; Su, L.; Cao, C.; Xu, L.; Zhong, D.; Xu, L.; Liu, X. The chalcone 2'-hydroxy-4' ' $^{\prime}$-dimethoxychalcone activates death receptor 5 pathway and leads to apoptosis in human nonsmall cell lung cancer cells. IUBMB Life 2013, 65, 533-543. [CrossRef] [PubMed]

59. Sumiyoshi, M.; Taniguchi, M.; Baba, K.; Kimura, Y. Antitumor and antimetastatic actions of xanthoangelol and 4-hydroxyderricin isolated from Angelica keiskei roots through the inhibited activation and differentiation of M2 macrophages. Phytomedicine 2015, 22, 759-767. [CrossRef] 
60. Shi, Y.; Wu, W.-Z.; Huo, A.; Zhou, W.; Jin, X.-H. Isobavachalcone inhibits the proliferation and invasion of tongue squamous cell carcinoma cells. Oncol. Lett. 2017, 14, 2852-2858. [CrossRef] [PubMed]

61. Yagura, T.; Motomiya, T.; Ito, M.; Honda, G.; Iida, A.; Kiuchi, F.; Tokuda, H.; Nishino, H. Anticarcinogenic compounds in the Uzbek medicinal plant, Helichrysum maracandicum. J. Nat. Med. 2008, 62, 174-177. [CrossRef]

62. Ramirez-Tagle, R.; Escobar, C.A.; Romero, V.; Montorfano, I.; Armisén, R.; Borgna, V.; Jeldes, E.; Pizarro, L.; Simon, F.; Echeverria, C. Chalcone-induced apoptosis through caspase-dependent intrinsic pathways in human hepatocellular carcinoma cells. Int. J. Mol. Sci. 2016, 17, 260-278. [CrossRef] [PubMed]

63. Marques, A.; Pereira, S.; Paiva, R.; Cavalcante, C.; Sudo, S.; Tinoco, L.; Moreira, D.L.; Guimaraes, E.; Sudo, R.; Kaplan, M.; et al. Hypoglycemic effect of the methanol flower extract of piper claussenianum and the major constituent $2^{\prime}, 6^{\prime}$-dihydroxy-4'methoxychalcone in streptozotocin diabetic rats. Indian J. Pharm. Sci. 2015, 77, 237-243.

64. Enoki, T.; Ohnogi, H.; Nagamine, K.; Kudo, Y.; Sugiyama, K.; Tanabe, M.; Kobayashi, E.; Sagawa, H.; Kato, I. Antidiabetic activities of chalcones isolated from a Japanese herb, Angelica keiskei. J. Agric. Food Chem. 2007, 55, 6013-6017. [CrossRef]

65. Lin, C.-T.; Senthil Kumar, K.J.; Tseng, Y.-H.; Wang, Z.-J.; Pan, M.-Y.; Xiao, J.-H.; Chien, S.-C.; Wang, S.-Y. Anti-inflammatory activity of flavokawain B from Alpinia pricei Hayata. J. Agric. Food Chem. 2009, 57, 6060-6065. [CrossRef] [PubMed]

66. Lin, Y.; Kuang, Y.; Li, K.; Wang, S.; Song, W.; Qiao, X.; Sabir, G.; Ye, M. Screening for bioactive natural products from a 67-compound library of Glycyrrhiza inflata. Bioorg. Med. Chem. 2017, 25, 3706-3713. [CrossRef]

67. Franceschelli, S.; Pesce, M.; Vinciguerra, I.; Ferrone, A.; Riccioni, H.; Patruno, A.; Grilli, A.; Felaco, M.; Speranza, L. Licocalchone-C extracted from Glycyrrhiza glabra inhibits lipopolysaccharide-interferon- $\gamma$ inflammation by improving antioxidant conditions and regulating inducible nitric oxide synthase expression. Molecules 2011, 16, 5720-5734. [CrossRef] [PubMed]

68. Daikonya, A.; Kitanaka, S.; Katsuki, S. Antiallergic agents from natural sources 9. Inhibition of nitric oxide production by novel chalcone derivatives from Mallotus philippinensis (Euphorbiaceae). Chem. Pharm. Bull. 2004, 52, 1326-1329. [CrossRef] [PubMed]

69. Zampini, I.C.; Vattuone, M.A.; Isla, M.I. Antibacterial activity of Zuccagnia punctata Cav. ethanolic extracts. J. Ethnopharmacol. 2005, 102, 450-456. [CrossRef] [PubMed]

70. Costa, G.; Endo, E.H.; Cortez, D.; Nakamura, T.; Nakamura, C.; Filho, B.D. Antimicrobial effects of Piper hispidum extract, fractions and chalcones against Candida albicans and Staphylococcus aureus. J. Med. Mycol. 2016, 26, 217-226. [CrossRef] [PubMed]

71. Oldoni, T.L.C.; Cabral, I.S.; d'Arce, M.A.R.; Rosalen, P.L.; Ikegaki, M.; Nascimento, A.M.; Alencar, S.M. Isolation and analysis of bioactive isoflavonoids and chalcone from a new type of Brazilian propolis. Sep. Purif. Technol. 2011, 77, 208-213. [CrossRef]

72. Kulkarni, R.R.; Tupe, S.G.; Gample, S.P.; Chandgude, M.G.; Sarkar, D.; Deshpande, M.V.; Joshi, S.P. Antifungal dimeric chalcone derivative kamalachalcone E from Mallotus philippinensis. Nat. Prod. Res. 2014, 28, 245-250. [CrossRef]

73. Jayasinghe, L.; Balasooriya, B.; Padmini, W.C.; Hara, N.; Fujimoto, Y. Geranyl chalcone derivatives with antifungal and radical scavenging properties from the leaves of Artocarpus nobilis. Phytochemistry 2004, 65, 1287-1290. [CrossRef]

74. Mohammed, M.M.; Hamdy, A.-H.A.; El-Fiky, N.M.; Mettwally, W.S.; El-Beih, A.A.; Kobayashi, N. Anti-influenza A virus activity of a new dihydrochalcone diglycoside isolated from the Egyptian seagrass Thalassodendron ciliatum (Forsk.) den Hartog. Nat. Prod. Res. 2014, 28, 377-382. [CrossRef] [PubMed]

75. Dao, T.T.; Nguyen, P.H.; Lee, H.S.; Kim, E.; Park, J.; Lim, S.I.; Oh, W.K. Chalcones as novel influenza A (H1N1) neuraminidase inhibitors from Glycyrrhiza inflata. Bioorg. Med. Chem. Lett. 2011, 21, 294-298. [CrossRef]

76. Uchiumi, F.; Hatano, T.; Ito, H.; Yoshida, T.; Tanuma, S.-I. Transcriptional suppression of the HIV promoter by natural compounds. Antivir. Res. 2003, 58, 89-98. [CrossRef]

77. Park, J.-Y.; Ko, J.-A.; Kim, D.W.; Kim, Y.M.; Kwon, H.-J.; Jeong, H.J.; Kim, C.Y.; Park, K.H.; Lee, W.S.; Ryu, Y.B. Chalcones isolated from Angelica keiskei inhibit cysteine proteases of SARS-CoV. J. Enzyme Inhib. Med. Chem. 2016, 31, 23-30. [CrossRef]

78. Borges-Argáez, R.; Balnbury, L.; Flowers, A.; Giménez-Turba, A.; Ruiz, G.; Waterman, P.G.; Peña-Rodríguez, L.M. Cytotoxic and antiprotozoal activity of flavonoids from Lonchocarpus spp. Phytomedicine 2007, 14, 530-533. [CrossRef]

79. Rodrigues, D.; Maniscalco, D.; Silva, F.; Chiari, B.; Castelli, M.; Isaac, V.; Cicarelli, R.; Lopez, S. Trypanocidal activity of flavokawin B, a component of Polygonum ferrugineum Wedd. Planta Med. 2017, 83, 239-244. [CrossRef]

80. Chen, M.; Theander, T.G.; Christensen, S.B.; Hviid, L.; Zhai, L.; Kharazmi, A. Licochalcone A, a new antimalarial agent, inhibits in vitro growth of the human malaria parasite Plasmodium falciparum and protects mice from P. yoelii infection. Antimicrob. Agents Chemother. 1994, 38, 1470-1476. [CrossRef]

81. Yenesew, A.; Induli, M.; Derese, S.; Midiwo, J.O.; Heydenreich, M.; Peter, M.G.; Akala, H.; Wangui, J.; Liyala, P.; Waters, N.C. Anti-plasmodial flavonoids from the stem bark of Erythrina abyssinica. Phytochemistry 2004, 65, 3029-3032. [CrossRef] [PubMed]

82. Sakagami, H.; Masuda, Y.; Tomomura, M.; Yokose, S.; Uesawa, Y.; Ikezoe, N.; Asahara, D.; Takao, K.; Kanamoto, T.; Terakubo, S. Quantitative structure-cytotoxicity relationship of chalcones. Anticancer Res. 2017, 37, 1091-1098. [PubMed]

83. da Silva Lima, D.C.; do Vale, C.R.; Véras, J.H.; Bernardes, A.; Pérez, C.N.; Chen-Chen, L. Absence of genotoxic effects of the chalcone (E)-1-(2-hydroxyphenyl)-3-(4-methylphenyl)-prop-2-en-1-one) and its potential chemoprevention against DNA damage using in vitro and in vivo assays. PLoS ONE 2017, 12, e0171224.

84. Emayavaramban, M.; Santhi, N.; Gopi, C.; Manivannan, C.; Raguraman, A. Synthesis, Characterization and anti-diabetic activity of 1,3, 5-triaryl-2-pyrazolines in acetic acid solution under ultrasound irradiation. Int. Lett. Chem. Phys. Astron. 2003, 9, 172-185. [CrossRef] 
85. Chinthala, Y.; Thakur, S.; Tirunagari, S.; Chinde, S.; Domatti, A.K.; Arigari, N.K.; Srinivas, K.; Alam, S.; Jonnala, K.K.; Khan, F. Synthesis, docking and ADMET studies of novel chalcone triazoles for anti-cancer and anti-diabetic activity. J. Med. Chem. Eur. 2015, 93, 564-573. [CrossRef] [PubMed]

86. Hsieh, H.-K.; Tsao, L.-T.; Wang, J.-P.; Lin, C.-N. Synthesis and anti-inflammatory effect of chalcones. J. Pharm. Pharmacol. 2000, 52, 163-171. [CrossRef] [PubMed]

87. Won, S.-J.; Liu, C.-T.; Tsao, L.-T.; Weng, J.-R.; Ko, H.-H.; Wang, J.-P.; Lin, C.-N. Synthetic chalcones as potential anti-inflammatory and cancer chemopreventive agents. J. Med. Chem. Eur. 2005, 40, 103-112. [CrossRef]

88. Jantan, I.; Bukhari, S.N.A.; Adekoya, O.A.; Sylte, I. Studies of synthetic chalcone derivatives as potential inhibitors of secretory phospholipase A2, cyclooxygenases, lipoxyugenase and pro-inflammatory cytokines. Drug Des. Dev. Ther. 2014, 8, 1405-1418. [CrossRef]

89. Chen, Y.-F.; Wu, S.-N.; Gao, J.-M.; Liao, Z.-Y.; Tseng, Y.-T.; Fulop, F.; Chang, F.-R.; Lo, Y.-C. The antioxidant, anti-inflammatory, and neuroprotective properties of the synthetic chalcone derivative AN07. Molecules 2020, 25, 2907. [CrossRef] [PubMed]

90. Solankee, A.; Kapadia, K.; Ana Ćirić, M.; Soković, I.; Doytchinova, A.; Geronikaki, A. Synthesis of some new S-triazine based chalcones and their derivatives as potent antimicrobial agents. J. Med. Chem. Eur. J. Med. Chem. 2010, 45, 510-518. [CrossRef] [PubMed]

91. Chu, W.-C.; Bai, P.-Y.; Yang, Z.-Q.; Cui, D.-Y.; Hua, Y.-G.; Yang, Y.; Yang, Q.-Q.; Zhang, E.; Qin, S. Synthesis and antibacterial evaluation of novel cationic chalcone derivatives possessing broad spectrum antibacterial activity. J. Med. Chem. Eur. 2018, 143, 905-921. [CrossRef] [PubMed]

92. Arif, R.; Rana, M.; Yasmeen, S.; Amaduddin, M.; Khan, M.S.; Abid, M.; Khan, M.S.; Rahisuddin, M. Facile synthesis of chalcone derivatives as antibacterial agents: Synthesis, DNA binding, molecular docking, DFT and antioxidant studies. J. Mol. Struct. 2020, 1208, 127905. [CrossRef]

93. Morao, L.G.; Lorenzoni, A.S.G.; Chakraborty, P.; Ayusso, G.M.; Cavalca, L.B.; Santos, M.B.; Marques, B.C.; Dilarri, G.; Zamuner, C.; Regasini, L.O.; et al. Investigating the modes of action of the antimicrobial chalcones BC1 and T9A. Molecules 2020, 25, 4596. [CrossRef]

94. Amole, K.L.; Bello, I.A.; Oyewale, A.O. Synthesis, characterization and antibacterial activities of new fluorinated chalcones. Chem. Afr. 2019, 2, 47-55. [CrossRef]

95. Xu, M.; Wu, P.; Shen, F.; Ji, J.; Rakesh, K.P. Chalcone derivatives and their antibacterial activities: Current development. Bioorg. Chem. 2019, 91, 103133. [CrossRef]

96. Zhang, M.; Prior, A.M.; Maddox, M.M.; Shen, W.-J.; Hevener, K.E.; Bruhn, D.F.; Lee, R.B.; Singh, A.P.; Reinicke, J.; Simmons, C.J.; et al. Pharmacophore modeling, synthesis, and antibacterial evaluation of chalcones and derivatives. ACS Omega 2018, 3 , 18343-18360. [CrossRef]

97. Sudhakar, C.; Suresj, J.; Valarmathi, N.; Sumathi, S.; Karthikeyan, A.; Arun, A. Synthesis, characterization of acrylate polymer having chalcone moiety: Evaluation of antimicrobial, anticancer and drug release study. J. Biomater. Sci. Polym. Ed. 2020, 32, 438-453. [CrossRef]

98. Lopez, S.N.; Castelli, M.V.; Zacchino, S.A.; Domínguez, J.N.; Lobo, G.; Charris-Charris, J.; Cortés, J.C.; Ribas, J.C.; Devia, C.; Rodríguez, A.M. In vitro antifungal evaluation and structure-activity relationships of a new series of chalcone derivatives and synthetic analogues, with inhibitory properties against polymers of the fungal cell wall. Bioorg. Med. Chem. 2001, 9, 1999-2013. [CrossRef]

99. Tailor, N.K. Synthesis and antifungal activity of certain chalcones and their reduction. Indo Glob. J. Pharm. Sci. 2014, 4, 25-28. [CrossRef]

100. Gupta, D.; Jain, D.K. Chalcone derivatives as potential antifungal agents: Synthesis and antifungal activity. J. Adv. Pharm. Technol. Res. 2015, 6, 114-117. [CrossRef]

101. Zheng, Y.; Wang, X.; Gao, S.; Ma, M.; Ren, G.; Liu, H. Synthesis and antifungal activity of chalcone derivatives. Nat. Prod. Res. 2015, 29, 1804-1810. [CrossRef] [PubMed]

102. Mellado, M.; Espinoza, L.; Madrid, A.; Mella, J.; Chavez-Weisser, E.; Diaz, K.; Cuellar, M. Design, synthesis, antifungal activity, and structure-activity relationship studies of chalcones and hybrid dihydrochromane-chalcones. Mol. Div. 2020, 24, 603-615. [CrossRef]

103. Andrade, J.T.; Santos, F.R.S.; Lima, W.G.; Sousa, C.D.F.; Oliveira, L.S.F.M.; Ribeiro, R.I.M.A.; Gomes, A.J.P.S.; Araujo, M.G.F.; Villar, J.A.F.P.; Ferreira, J.M.S. Design, synthesis, biological activity and structure activity relationship studies of chalcone derivatives as potential anti-Candida agents. J. Antibiot. 2018, 71, 702-712. [CrossRef] [PubMed]

104. Lagu, S.B.; Yejella, R.P.; Bhandare, R.R.; Shaik, A.B. Design, synthesis, and antibacterial and antifungal activities of novel trifluoromethyl and trifluoromethoxy substituted chalcone derivatives. Pharmaceuticals 2020, 13, 375. [CrossRef] [PubMed]

105. Cole, A.L.; Hossain, S.; Cole, A.M.; Phanstiel, O. Synthesis and bioevaluation of substituted chalcones, coumaranones and other flavonoids as anti-HIV agents. Bioorg. Med. Chem. 2016, 24, 2768-2776. [CrossRef] [PubMed]

106. Mateeva, N.; Eyunni, S.V.; Redda, K.K.; Ononuju, U.; Hansberry II, T.D.; Aikens, C.; Nag, A. Functional evaluation of synthetic flavonoids and chalcones for potential antiviral and anticancer properties. Bioorg. Med. Chem. Lett. 2017, 27, 2350-2356. [CrossRef]

107. Fu, Y.; Zeng, L.H.; Ren, X.; Song, B.; Hu, D.; Gan, X. New chalcone derivatives: Synthesis, antiviral activity and mechanism of action. RSC Adv. 2020, 10, 24483. [CrossRef] 
108. Elkhalifa, D.; Al-Hashimi, I.; Al Moustafa, A.-E.; Khalil, A. A comprehensive review on the antiviral activities of chalcones. J. Drug Target. 2021, 29, 403-419. [CrossRef] [PubMed]

109. Marinov, R.; Markova, N.; Krumova, S.; Yotovska, K.; Zaharieva, M.M.; Genova-Kalou, P. Antiviral properties of chalcones and their synthetic derivatives: A mini review. Pharmacia 2020, 67, 325-337. [CrossRef]

110. Zhou, D.; Xie, D.; He, F.; Song, B.; Hu, D. Antiviral properties and interaction of novel chalcone derivatives containing a purine and benzenesulfonamide moiety. Bioorg. Med. Chem. Lett. 2018, 28, 2091-2097. [CrossRef] [PubMed]

111. Wang, J.; Huang, L.; Cheng, C.; Li, G.; Xie, J.; Shen, M.; Chen, Q.; Li, W.; He, W.; Qiu, P.; et al. Design, synthesis and biological evaluation of chalcone analogues with novel dual antioxidant mechanisms as potential anti-ischemic stroke agents. Acta Pharm. Sin. B 2019, 9, 335-350. [CrossRef]

112. Stepanic, V.; Matijasic, M.; Horvat, T.; Verbanac, D.; Kucerova-Chlupacova, M.; Saso, L.; Zarkovic, N. Antioxidant activities of alkyl substiyuted pyrazine derivatives of chalcones-In vitro and in silico study. Antioxidants 2019, 8, 90. [CrossRef]

113. Lahsasni, A.S.; Al Korbi, F.H.; Aljaber, N.A.-A. Synthesis, characterization and evaluation of antioxidant activities of some novel chalcones analogues. Chem. Cent. J. 2014, 8, 32. [CrossRef] [PubMed]

114. Al Zahrani, N.A.; El-Shishtawy, R.M.; Elaasser, M.M.; Asiri, A.M. Synthesis of novel chalcone-based phenothiazine derivatives as antioxidant and anticancer agents. Molecules 2020, 25, 4566. [CrossRef]

115. Wu, J.-Z.; Cheng, C.-C.; Shen, L.-L.; Wang, Z.-K.; Wu, S.-B.; Li, W.-L.; Chen, S.-H.; Zhou, R.-P.; Qiu, P.-H. Synthetic chalcones with potent antioxidant ability on H2O2-induced apoptosis in PC12 cells. Int. J. Mol. Sci. 2014, 15, 18525-18539. [CrossRef] [PubMed]

116. Venkatachalam, H.; Nayak, Y.; Jayashree, B.S. Synthesis, characterization and antioxidant activities of synthetic chalcones and flavones. APCBEE Procedia 2012, 3, 209-213. [CrossRef]

117. Padhye, S.; Ahmad, A.; Oswa, N.; Sarkar, F.H. Emerging role of garcinol, the antioxidant chalcone from Garcinia indica Choisy and its synthetic analogs. J. Hematol. Oncol. 2009, 2, 38. [CrossRef] [PubMed]

118. Iqbal, H.; Prabhakar, V.; Sangith, A.; Chandrika, B.; Balasubramanian, R. Synthesis, anti-inflammatory and antioxidant activity of ring-A-monosubstituted chalcone derivatives. Med. Chem. Res. 2014, 23, 4383-4394. [CrossRef]

119. Ugwu, D.I.; Ezema, B.E.; Eze, F.U.; Onoabedje, E.A.; Ezema, C.G.; Ekoh, O.C.; Ayogu, J.I. Synthesis and antimalarial activities of chalcone derivatives. Int. J. ChemTech Res. 2015, 7, 1966-1984.

120. Yadav, N.; Dixit, S.K.; Bhattacharya, A.; Mishra, L.C.; Sharma, M.; Awasthi, S.K.; Bhasin, V.K. Antimalarial activity of newly synthesized chalcone derivatives in vitro. Chem. Biol. Drug Des. 2012, 80, 340-347. [CrossRef]

121. Dominguez, J.N.; de Dominguez, N.G.; Rodrigues, J.; Acosta, E.M.; Caraballo, N.; Leon, C. Synthesis and antimalarial activity of uranyl Bis-chalcone in vitro and in vivo. J. Enzyme Inhib. Med. Chem. 2013, 28, 1267-1273. [CrossRef]

122. Roussaki, M.; Hall, B.; Lima, S.C.; da Silva, A.C.; Wilkinson, S.; Detsi, A. Synthesis and anti-parasitic activity of a novel quinolinone-chalcone series. Bioorg. Med. Chem. Lett. 2013, 23, 6436-6441. [CrossRef]

123. Sinha, S.; Radotra, B.D.; Medhi, B.; Batovska, D.I.; Markova, N.; Sehgal, R. Ultrastructural alterations in Plasmodium falciparum induced by chalcone derivatives. BMC Res. Notes 2020, 13, 290. [CrossRef] [PubMed]

124. Gonzalez, L.A.; Upegui, Y.A.; Rivas, L.; Echeverri, F.; Escobar, G.; Robledo, S.M.; Quinones, W. Effect of substituents in the A and B rings of chalcones on antiparasitic activity. Arch. Pharm. 2020, 353, 2000157. [CrossRef] [PubMed]

125. Bhoj, P.; Togre, N.; Bahekar, S.; Goswami, K.; Chandak, H.; Patil, M. Immunomodulatory activity of sulfonamide chalcone compounds in mice infected with filarial parasite Brugia malayi. Indian J. Clin. Biochem. 2019, 34, 225-229. [CrossRef]

126. Lee, J.S.; Bukhari, S.N.A.; Fauzi, N.M. Effects of chalcone derivatives on players of the immune system. Drug Des. Dev. Ther. 2015, 9, 4761-4778.

127. Arshad, L.; Jantan, I.; Bukhari, S.N.A.; Haque, M.A. Immunosuppressive effects of natural $\alpha, \beta$-unsaturated carbonyl-based compounds and their analogs and derivatives on immune cells: A review. Front. Pharmacol. 2017, 8, 22. [CrossRef] [PubMed] 